\title{
A Simulation Analysis for LED Spatial Distribution for Indoor Visible Light Communication
}

Ala Khalifeh ( $\square$ ala.khalifeh@gju.edu.jo )

German Jordanian University https://orcid.org/0000-0003-3600-8090

\section{Karthikeyan Alakappan}

SSN College of Engineering: Sri Sivasubramaniya Nadar College of Engineering

\section{Barath kumar}

SSN College of Engineering: Sri Sivasubramaniya Nadar College of Engineering

Jayanth Prabakaran

SSN College of Engineering: Sri Sivasubramaniya Nadar College of Engineering

\section{Prabagarane Nagaradjane}

SSN College of Engineering: Sri Sivasubramaniya Nadar College of Engineering

\section{Manuscript}

Keywords: Visible light communication, light engineering, BER

Posted Date: February 9th, 2021

DOI: https://doi.org/10.21203/rs.3.rs-193050/v1

License: (c) (1) This work is licensed under a Creative Commons Attribution 4.0 International License. Read Full License

Version of Record: A version of this preprint was published at Wireless Personal Communications on August 25th, 2021. See the published version at https://doi.org/10.1007/s11277-021-08972-5. 
A Simulation Analysis for LED Spatial Distribution for Indoor Visible Light Communication

\author{
Ala' F. Khalifeh ${ }^{1}$, Karthikeyan Alakappan ${ }^{2}$, Barath Kumar $^{2}$, Jayanth Prabakaran²,Prabagarane Nagaradjane ${ }^{2}$ \\ ${ }^{1}$ Electrical and Communication Engineering Dept., German Jordanian University, Jordan \\ e-mail: ala.khalifeh@gju.edu.jo \\ ${ }^{2}$ Department of Electronics and communication engineering,
}

Sri Sivasubramaniya Nadar College of Engineering Chennai, India

email:karthikeyan16048@ece.ssn.edu.in, jayanth16045@ece.ssn.edu.in, barath16023@ece.ssn.edu.in, prabagaranen@ssn.edu.in

\begin{abstract}
Visible light communication (VLC) is a promising technology that can jointly be used to accomplish the typical lighting functionalities of the light-emitting diodes (LEDs) and data transmission, where light intensity can be modulated on a high rate that cannot be noticed by the human eye. In this paper, a VLC simulation framework to study the effect of LEDs' distributions on different room dimensions is proposed by considering the performance metrics such as light intensity quality in accordance with the International Organization for Standardization (ISO) recommendation, and data transmission efficiency measured in terms of bit error rate (BER). To achieve the abovementioned performance metrics, a VLC communication system is designed that modulates the data, transmits it over the room utilizing the communication channel that is modeled using an accurate ray-tracing algorithm, and receives it by exploiting different receivers that are uniformly distributed in the room. Our work is different from the other published works which either studied the data transmission efficiency or lighting quality but not both. Consequently, this study can be used as a methodological foundation to design an efficient VLC that satisfies the ISO lighting requirement, application-specific BER and quality. Furthermore, a video transmission use case has been demonstrated which shows how the video quality can be significantly improved when increasing the number of transmitters, thus justifying the need for increasing the number of transmitters in scenarios that involves video transmission in an indoor VLC environment.
\end{abstract}

KEYWORDS: Visible light communication; light engineering; BER

\title{
1. INTRODUCTION
}

Nowadays, wireless networks have seen an unprecedented demand for increased data rate requirements. To facilitate realistic coverage with the data rate requirements, a considerable bandwidth is needed which remains a limiting factor due to the scantiness of radio frequency spectrum which bears the entire load of commercial and noncommercial wireless transmission applications. Most wireless communication systems are based on RF communication technologies. These applications are numerous, that range from maritime radio navigation to satellite communications. The advancement in data streaming and multimedia quality has an adverse effect on the available radio spectrum, which is soon set to hit a roadblock. Consequently, of late, researchers across academia and industries have started exploring alternate wireless transmission technologies to meet the ever-increasing demand. The ultraviolet spectrum is too dangerous for humans to be used for commercial purposes. The infrared spectrum also proves to be futile for commercial wireless 
transmission due to the power limitations induced to ensure safety to the human eyes. In this context, the huge bandwidth available in the unlicensed electromagnetic spectrum in the optical domain is seen as a promising solution to the spectrum crunch. In view of this, research on wireless optical communications has seen an upsurge interest in the past decade [1]. Visible light communication (VLC) makes use of the higher frequencies in the visual band and extends the capabilities of data transmission using general light sources. It transmits data by high-speed switching or flickering at a rate that is not perceivable to the naked eye. VLC has been regarded as an appealing communication technology to fulfill the high data rate demands and as a new affiliate in the beyond fifth-generation $(5 \mathrm{G})$ heterogeneous networks (HetNets). As 5G networks are being deployed, VLC can aid in the design of systems that can serve as a hybrid in utilizing both the radio and visible spectrum to facilitate the high-speed data transmission without putting much strain on the radio spectrum.

Additionally, significant research had been devoted to the optical spectrum specifically in the infrared region $[2,3]$. Furthermore, advancements in illumination technology coupled with research efforts on high data rate have made VLC indoor optical wireless communications a reality [4]. The use of light-emitting diodes (LEDs)-based transmitter and photo detector-aided receivers that are of low cost and highly energyefficient when used in VLC offers significant advantages in both lighting and wireless communications. An LED lighting equipment is easy to install and safe to the human eye. LEDs are available in different shapes to aid specific illumination and communication aspects, offer good modulation performance, longer life span, and excellent brightness. Moreover, the low-energy consumption and high-speed data communication that can be achieved by using LEDs along with the exploitation of huge bandwidth available in the unlicensed spectrum results in a new paradigm for data transmission. Besides, energy-efficient illumination and lighting functionality can also be attained.

Apart from the usage of the visible light spectrum by the VLC system, it offers several other advantages. To be specific, it offers secured communication as the light cannot penetrate through walls and other opaque objects. This provides protection against eavesdroppers and illegal tapping of data. To receive the data, the user or the receiver has to be in the field of view of LEDs, which will be noticed by the users in the room. The VLC system is also less hazardous to human health. In comparison to the infrared and ultraviolet communication, a VLC system can be implemented more comfortably and, consequently, a higher transmission power can be used to improve the communication link quality. In addition, VLC in the visible light spectrum is different from the RF spectrum and does not interfere with the existing RF communication devices [1]. This allows the VLC system to easily satisfy the electromagnetic compatibility requirements, and hence, VLC systems can be used in the applications, which are prone to electromagnetic interference such as hospitals, airplanes, and chemical plants. VLC can also be used in many application scenarios such as underwater communications where short radio waves cannot penetrate through long distances in water and as indoor positioning systems for departmental stores. Also, the huge indoor setups that require directions for efficient movement may use LED arrays placed few meters apart through the indoor setup. It also finds an interesting application in the automotive industry in the form of vehicular VLC, which enables the communication between cars, traffic signals, and road signs. Cooperative RF-VLC communications finds application in a typical cellular network, device-to-device communications, and many other applications and scenarios as explained in [5-7].

The rest of the paper is organized as follows: Section 2 presents the most related work in the literature. Section 3 describes the indoor VLC system model. Section 4 presents the LED spatial distribution simulation setup. Section 5 presents the simulation results and performance evaluation. Finally, section 6 concludes the paper and presents future work. 


\section{LITERATURE REVIEW}

In [8], Cheng et al. presented a ray-tracing algorithm based on wavelength for modeling multisource indoor channel impulse response for VLC, and demonstrated that blue LED exhibits a larger bandwidth in a plastic wall room. In particular, this research contribution has analyzed the root-mean-square (RMS) delay spread and average delay for three wavelengths. This work has illustrated that blue LED has larger bandwidth than other wavelengths. By contrast, in [9], authors have employed white LEDs for not only illuminating the rooms but also realizing VLC for indoor communications. Furthermore, with the help of numerical analysis, the influence of reflection and interference has been studied. A multi-user multi-input single-output (MUMISO) VLC broadcast is delved into with zero forcing (ZF) and ZF-dirty paper precoding techniques and biasing model for an indoor scenario [10]. The authors here have addressed the optimization problems in order to maximize the throughput subjected to the optical transmit power constraint. In the research article [11], authors have addressed a few basic techniques and key issues related to VLC realization. In [12], a MATLAB ${ }^{\circledR}$-based simulation study has been reported which studies the distributions of illuminance and RMS delay-spread indoor VLC landscape. The simulation program considers the transmitters' positions and reflections at each of the walls for an indoor VLC system. The authors in [13] have realized a digital transmission with the aid of RS-485 protocol. More specifically, the authors have exploited off-the-shelf white LED and demonstrated an indoor wireless VLC system that provides a coverage of $2.5 \mathrm{~m}$ and 7-10\% bit error rate (BER). Zeyu et al. [14] have presented a new indoor VLC prototype that uses diffuse links to achieve acceptable data rates. This VLC system also supports mobility under line of sight (LOS) while at the same time, aiding illumination and transmission in excess of several meters. Additionally, it has been demonstrated that this VLC system is capable of delivering data rate that is in excess of $1 \mathrm{Mbps}$.

In [15], the impact of different modulation schemes including multiple pulse position modulation is discussed. It also explores the performance of the different modulation schemes for VLC by comparing the $\mathrm{BER}$, signal-to-noise ratio (SNR) and data rate. The main aim is to perform the flickering improvement and dimming sustainability to offer optimal data rates for communication. The contribution in [16] reports a new constellation design called space-collaborative constellation for an indoor multi-input multi-output (MIMO) VLC. This new design has been shown to provide better BER under adverse operating condition than the conventional approaches that employ repetition code, spatial modulation and spatial multiplexing. In the research paper [17], the scope of the VLC to serve as a complementary technology to the current radio frequency standards is discussed. The paper includes a comprehensive survey of VLC, as well as the main concepts and challenges related to this emerging area. In [18] and [19], we have studied experimentally an indoor VLC system. In particular, in these contributions we have implemented, tested, and evaluated an indoor VLC. In [20], the performance of an indoor VLC system with randomly deployed LEDs is compared with fixed geometries such as circular and square. The SNR profile inside the room that changes with respect to LED placement as well as receiver's position is compared between different arrangements. Further, in [21], we have evaluated the potential utilization of VLC for car-to-car communication, and studied the effect of shadowing on VLC performance in [22]. Recently, we investigated the effect of shifting the LEDs transmitter and receivers positions on the communication performance in [23].

This work extends our previous works that aimed at designing and implementing a realistic yet efficient VLC transmission system. To be specific, against the background available in the open literature and our earlier work reported in [23], the main contributions of this paper are summarized as follows:

- We present an expanded simulation analysis where the effect of the number of transmitters and receivers' LEDs as well as their positions for different room sizes have been simulated and studied.

- Furthermore, a realistic non-LOS channel model has been utilized in the simulation which gives a more 
realistic results more than ever when compared with most of the presented works in the literature that utilize a LOS channel model.

- In addition, the illumination analysis for the LEDs' positions has been presented, thus giving a comprehensive study for both data transmission efficiency and light illumination quality.

- A video transmission use case over VLC utilizing different number of transmitters has been demonstrated. The simulation results showed that when video is transmitted over VLC, it is paramount to increase the number of transmitters to keep high-quality video transmission, thus justifying the high number of transmitters.

\section{INDOOR VLC SYSTEM}

A typical VLC system is depicted in Fig. 1. In what follows, a brief description of each block is provided.

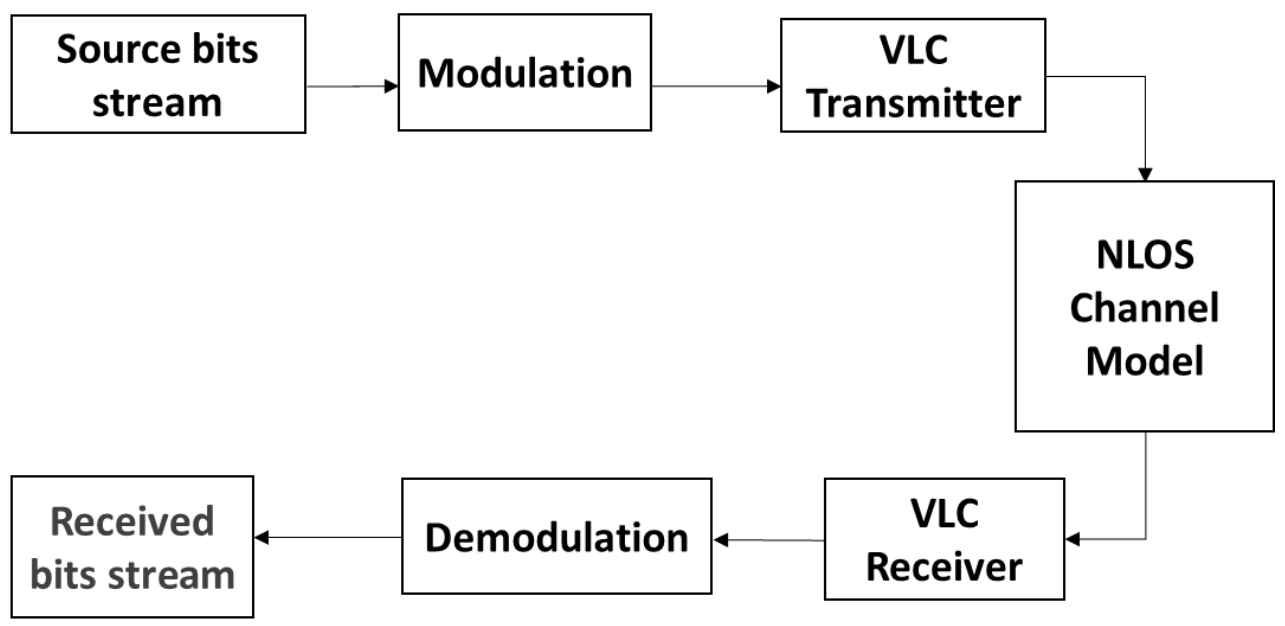

Fig. 1 A block diagram of a VLC system.

\subsection{Bit Stream Modulation}

As depicted in Fig. 1, the data bit stream is input to a modulator where an ON-OFF KEYING (OOK) modulation is utilized. There are a number of modulation schemes, which can be used for VLC. A bit one is simply represented by an optical pulse that occupies the entire or part of the bit duration, while a bit zero is represented by the absence of an optical pulse. Both the return-to-zero (RZ) and non-return-to-zero (NRZ) schemes can be applied. In the paper, the OOK NRZ is used although it yields relatively low data rate compared to other modulation schemes, but the BER performance is the best for OOK modulation compared to other modulation schemes [24], which makes it a good candidate for applications that require high reliability than high data rates.

\subsection{VLC Transmitter}

In VLC, LEDs play the role of transmitters along with its usual role as a lighting device. The LEDs are currently the best transmitters due to its long lifetime, cost effectiveness and energy efficiency. LEDs are normally modeled as Lambertian source where the luminance is distributed uniformly in all directions, 
whereas the luminous intensity is different in all directions. The luminous intensity for a Lambertian source is given by Equation (1) [16].

$$
I(\varphi)=I(0) \cos (\varphi)^{n}
$$

where $\varphi$ is the angle of irradiance, $I(0)$ is the center luminance intensity that corresponds to the case, where $\varphi=0$, and $n$ is the Lambert index calculated using Equation (2) [16].

$$
n=-\frac{\ln 2}{\ln \cos \varphi_{1 / 2}} \ldots \ldots \ldots \ldots \ldots \ldots
$$

Furthermore, Figure 2 depicts the main parameters used in characterizing an LED source and a photodiode receiver such as the field-of-view (FOV), and $\varphi_{1 / 2}$ is the transmitter's semi-angle at half-power.

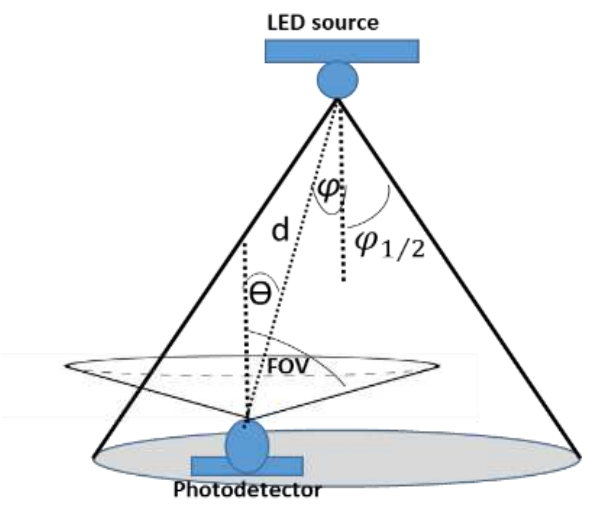

Fig. 2 The main parameters used in characterizing an LED source and a photo-diode receiver [16].

\subsection{NLOS Channel Model}

Two channels types are commonly used to model a VLC system. The first one is the LOS model (LOS), while the other is the non-LOS model (NLOS) or sometime called as the diffused link channel model [24]. In case of the LOS channel, the transmitter and receiver communicate through direct LOS transmission using narrow beams, where no wall or surrounding reflections are considered. This type of channel can be used for high transmission rates. However, this type of transmission faces shadowing effect and blocking, and may not resemble realistic scenarios where light beams undergo different paths and reflections before reaching the receiver. NLOS channel on the other side, takes into consideration the possible reflections of the light beams from the walls and surrounding environments and hence it is considered more realistic and practical. Clearly, this type of channel experiences multipath effect due to multiple reflections, but it does not experience the shadowing effect like the LOS channel. In this paper, we will be considering the NLOS channel for the simulation analysis and implement it using the modified Monte Carlo ray-tracing algorithm (MMC) proposed in [21].

\subsection{Channel Impulse Response Estimation}

To generate a VLC indoor channel impulse response, the MMC algorithm is used. Several rays are generated from the emitter position with a distribution probability that equals to the emission profile or the angular optical intensity function. The rays strike the obstacles in the path and the point where it strikes the obstacle is taken as an optical source from where a new ray is generated because LEDs are considered Lambertian sources. The process continues until the time of flight. After every reflection, the power of the 
ray is decreased by the reflection coefficient of the obstacle. When a ray strikes a point, a new ray is generated and the reflected power contribution towards the receiver is calculated. In this paper, three reflections are considered as they contribute to most of the reflected power and rest of the reflected power contribution is ignored, as higher order reflections are negligible. The process continues for the number of rays generated from the source. Figure 3 shows a sample NLOS impulse response in time and frequency domains for an indoor room with a single transmitter and receiver located at $[3.5,3.5,3],[0.5,1,3] \mathrm{m}$, respectively, in a room of dimension $[7,7,3] \mathrm{m}$.

\subsection{VLC Receiver}

The light pulse is transmitted through a multi-path diffuse channel and received at the photo-detector. The received signal is passed through a matched filter. The detected signal at the input of the matched filter is given by Equation (3).

$$
i(t)=I_{p} * h(t)+n(t)
$$

where $h(t)$ is the NLSO channel impulse response which is estimated by the MMC algorithm, $n(t)$ is the additive white Gaussian noise due to shot noise, ambient light and thermal noise. $I_{\mathrm{p}}$ is the peak photocurrent [25]. The received bits are then demodulated and the performance in terms of BER is evaluated for different LEDs transmitters, numbers, positions, and rooms' dimensions as will be explained in the next section.

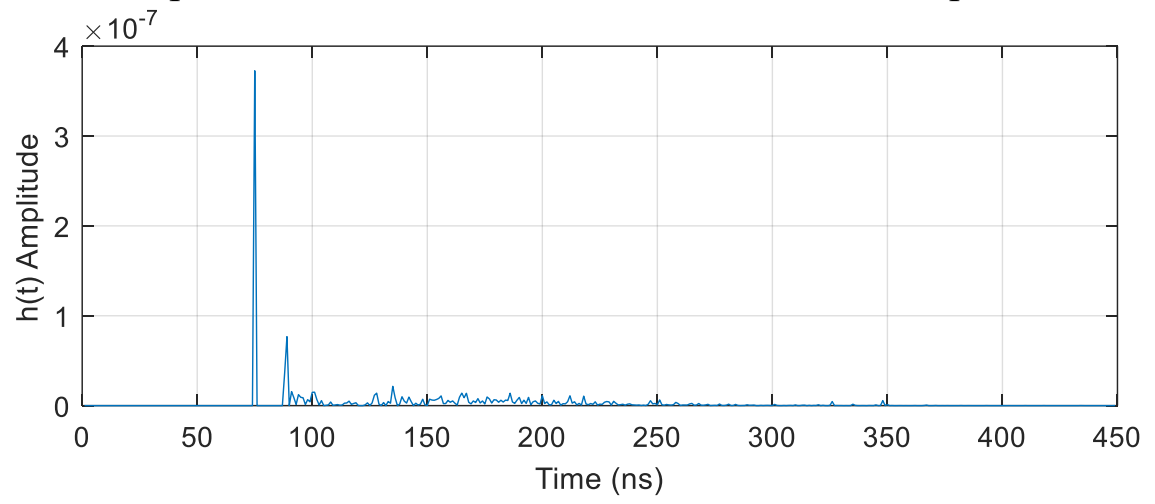

(a)

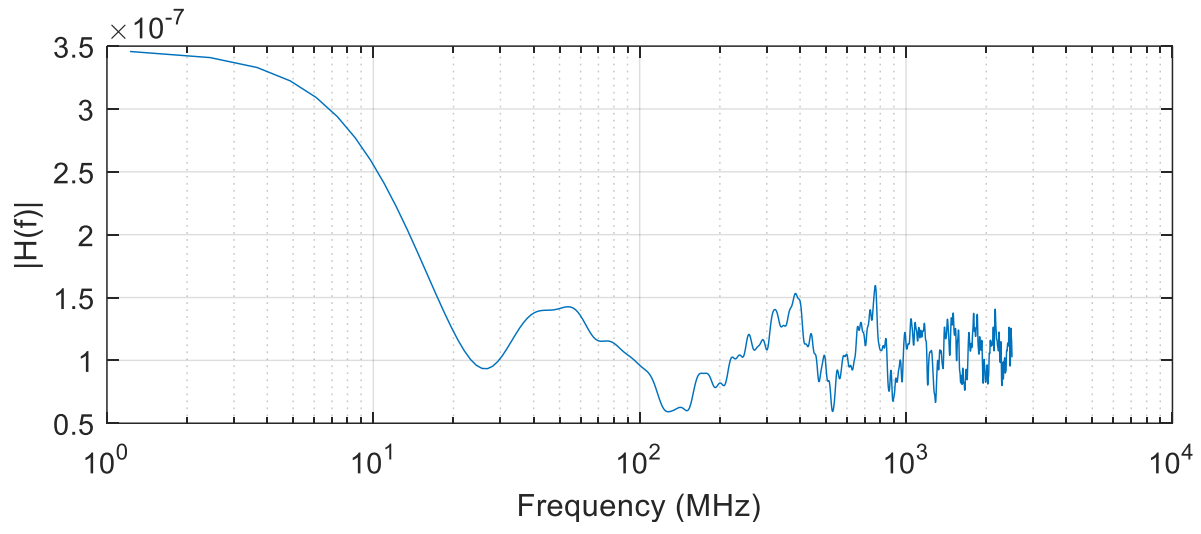

(b)

Fig. 3 A NLOS impulse response in (a) time and (b) frequency domains for an indoor room of a single transmitter and receiver located at $[3.5,3.5,3],[0.5,1,3] \mathrm{m}$, respectively, in a room dimension of $[7,7,3] \mathrm{m}$. 


\section{LED SPATIAL DISTRIBUTION AND ILLUMINATION EVALUATION}

In order to realize the performance of VLC for different LED spatial distributions, different scenarios have been considered where the number of transmitters, their positions, the room dimensions has been varied. Furthermore, the number of rays used in modeling and simulating the LED, which is noticed to be affecting the performance of the system has been studied as well. A grid arrangement of LEDs has been used which is achieved by dividing the room into equal grids both horizontally and vertically depending on the number of LED transmitters, where one transmitter is allocated to each grid. Different room dimensions have been investigated as will be depicted in the simulation section. In order to simulate the grid arrangement, two mesh-grid functions in MATLAB ${ }^{\circledR}$ tool have been used to simulate the transmitter and the receiver planes. On the transmitter mesh-grid, the positions of the ceiling lamp arrays are set. On the other hand, the positions of the receivers are not fixed. Since the received light across the entire space is being measured, the receiver mesh-grid is assumed a continuous plane of photodiodes, with each point on the grid representing the received power of a photodiode at that location. However, due to the limitations of the simulation, the "continuous" plane of photodiodes is a set of discrete points on the mesh-grid. The resolution of the receiver mesh-grid could be increased at the cost of longer simulation runtime. To measure the effect of every array at each point on the receiver plane, the distance between each transmitter and every point on the receiver plane needs to be calculated as seen in Fig. 4. The distance is measured by using Equation (4).

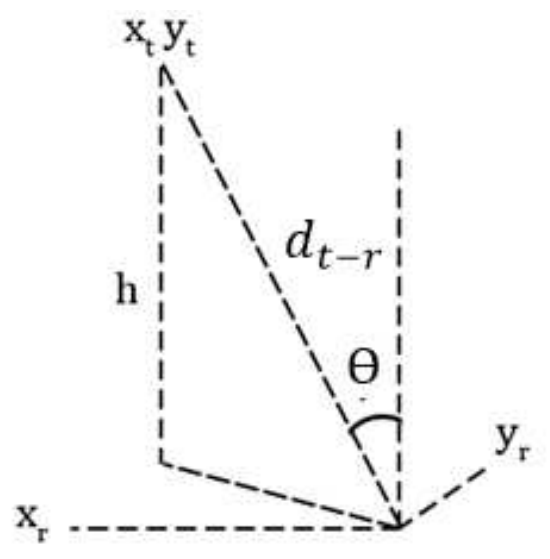

Fig. 4 Assuming a fixed transmitter at $x_{t}, y_{t}$, the distance between that transmitter and any point on the receiver $x_{r}, y_{r}$ mesh-grid can be calculated using Pythagoras' theorem.

$$
d_{t-r}=\sqrt{\left(x_{r}-x_{t}\right)^{2}+\left(y_{r}-y_{t}\right)^{2}+h^{2}} \ldots \ldots \ldots
$$

where $d_{t-r}$ is the distance between the transmitter and receiver. Following the calculation of the distance, the measurement of the angle between the normal to the receiver mesh-grid and the LOS path needs to be calculated. This is calculated using the simple trigonometry as depicted in Equation (5).

$$
\theta=\cos ^{-1}\left(\frac{h}{d_{t-r}}\right)
$$

To calculate the channel gain $(G)$, we use Equation (6). 


$$
G=\frac{(n+1) \times D A \times \cos (\varphi)^{(n+1)}}{2 \pi \times d_{t-r}^{2}}
$$

DA is the photo-detector area. The calculation of the received power $\left(P_{\mathrm{r}}\right)$ must take into account the remaining parameters set in the beginning of the simulation for the additional optional components. The power transmitted $\left(P_{\mathrm{t}}\right)$, the optical concentrator gain $(C)$, the optical filter gain $(F)$ as well as the calculated channel gain are all considered in Equation (7).

$$
P_{r}=P_{t} \times G \times C \times F
$$

The received power has a direct effect on the performance of VLC. For instance, if we use a non-return-tozero on-off keying (NRZ-OOK) modulation scheme, then the probability of the error is given by Equation (8) [10]:

$$
p(e)=Q(\sqrt{\mathrm{SNR}})
$$

Where $S N R=\frac{P_{r}}{P n}$, where $P_{n}$ is the noise power at the receiver. Finally, the photodiodes placed far enough from the light source to have a receiver angle higher than that of the initially set FOV value are assumed to receive no light. Therefore, a "find" function in MATLAB ${ }^{\circledR}$ is called to find all the photodiodes with receivers' angles, which exceed the FOV angle to set their respective received power to zero. Using the matrix properties in MATLAB ${ }^{\circledR}$, all the previously mentioned calculations can be performed for every point on the receiver mesh-grid. The resulting matrix would contain the power distribution because of single array of LEDs at a single point, i.e., one ceiling lamp. In order to evaluate the effectiveness of the grid arrangement on the illuminance intensity, the illuminance due to the positioning of LEDs is evaluated using the horizontal illumination $E_{h}$ given by Equation (9) [23].

$$
E_{h}=I(0) \cos ^{n} \varphi / d_{t-r}^{2} \cdot \cos \theta
$$

where $I(0)$ is the center luminance intensity.

\section{SIMULATION ANALYSIS}

Several experiments have been conducted to analyze the effect of changing the number of LEDs transmitters and their locations for various room dimensions. The main target of the simulation analysis is to study both the LEDs' illumination efficiency and performance of the VLC systems. MATLAB ${ }^{\circledR}$ is used to generate the simulation results. The simulation code is a modified version of the code presented in [12]. The main simulation parameters used in this work is depicted in Table 1.

\subsection{LEDs’ Illumination Performance Evaluation}

An essential functionality of the VLC system is to provide efficient lighting. Hence, the LEDs' illumination efficiency for different scenarios has been investigated. According to the International Organization for Standardization (ISO), the illuminance has to be between $300 l_{\mathrm{x}}$ and 1,500 $l_{\mathrm{x}}$ for office work [27]. 
Table 1 VLC main simulation parameters

\begin{tabular}{|c|c|}
\hline Parameter & Value/equation \\
\hline$\varphi_{1 / 2}$ & $70^{\circ}$ \\
\hline PLED & $\begin{array}{c}\text { Transmitted optical power by individual LED } \\
\text { equals to } 20 \mathrm{dBm}\end{array}$ \\
\hline $\begin{array}{l}\text { NLED: Number of } \\
\text { LEDs. } \\
\text { Note: Number of LED } \\
\text { array }= \\
\text { NLED } \times \text { NLED }\end{array}$ & 60 \\
\hline $\begin{array}{l}P t: \text { total transmitted } \\
\text { power }\end{array}$ & NLED $\times$ NLED $\times$ PLED \\
\hline DA & $10-4 \mathrm{~m}$ \\
\hline $\begin{array}{l}\text { Ts: gain of an optical } \\
\text { filter }\end{array}$ & 1 \\
\hline $\begin{array}{c}\text { Index: refractive index of } \\
\text { a lens at a PD }\end{array}$ & 1.5 \\
\hline FOV & $70^{\circ}$ \\
\hline $\begin{array}{c}l_{x}: l_{y}: l_{z} ; \\
\text { room dimensions in } \\
\text { meter }\end{array}$ & $5: 5: 3 \mathrm{~m}$ \\
\hline $\begin{array}{c}h: \text { the distance between } \\
\text { the source and receiver } \\
\text { plane }\end{array}$ & $2.15 \mathrm{~m}$ \\
\hline$\theta$ & $30^{\circ}$ \\
\hline$n$ & 1 \\
\hline $\begin{array}{l}\text { Central luminance } \\
\text { intensity }\left(I_{0}\right)\end{array}$ & $0.73 l_{x}$ \\
\hline Total luminance intensity & $60 \times 60 \times I_{0}$ \\
\hline
\end{tabular}


Table 2 LEDs grid arrangement

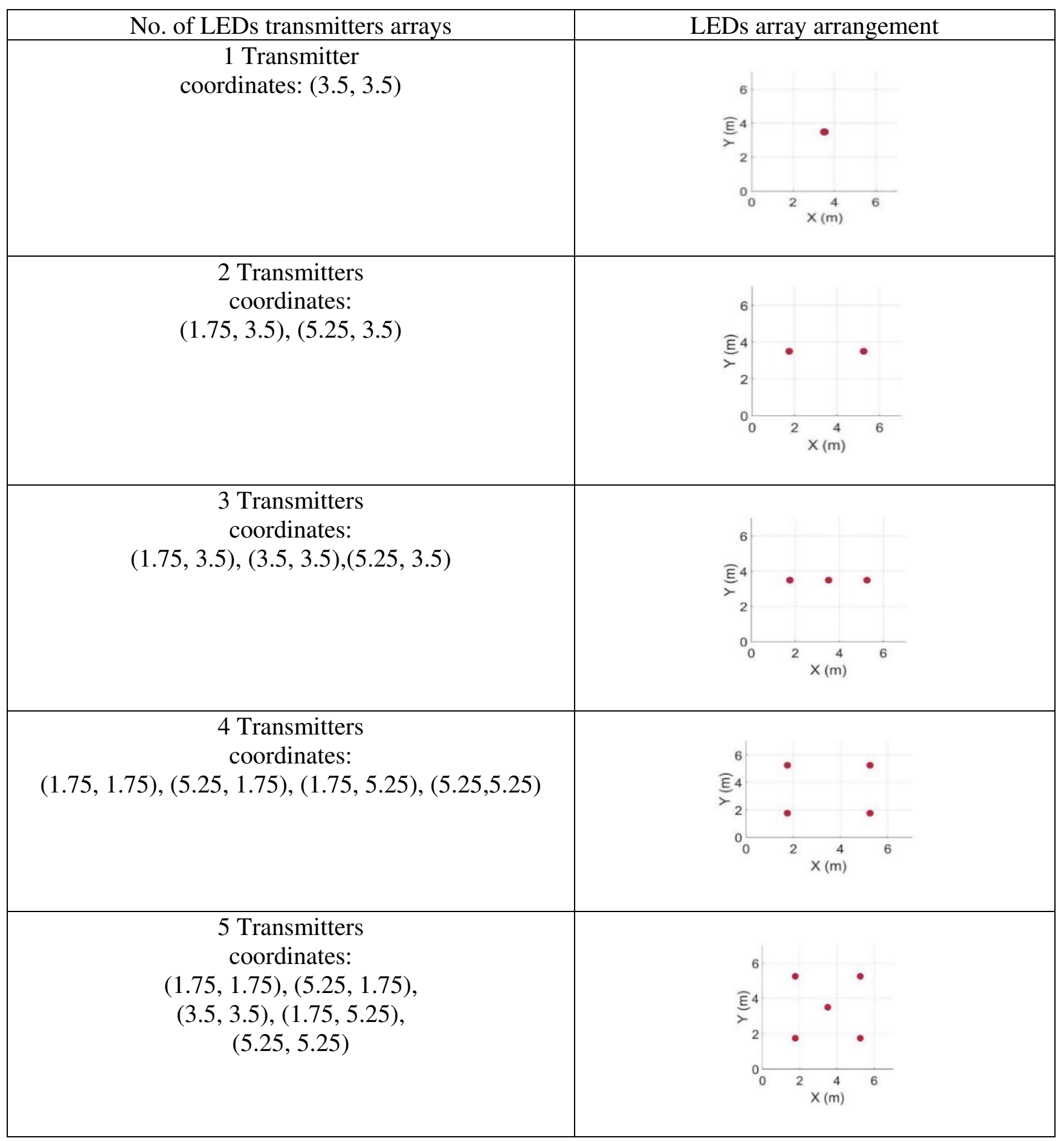




\begin{tabular}{|c|c|}
\hline $\begin{array}{c}6 \text { Transmitters } \\
\text { coordinates: } \\
(1.75,1.75),(3.5,1.75) \\
(5.25,1.75),(1.75,5.25),(3.5,5.25),(5.25,5.25)\end{array}$ & 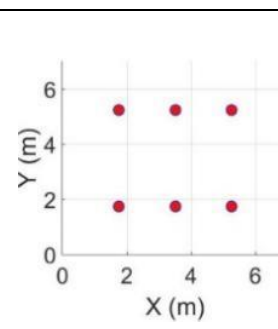 \\
\hline $\begin{array}{c}7 \text { Transmitters } \\
\text { coordinates: } \\
(1.75,1.75),(3.5,1.75), \\
(5.25,1.75),(3.5,3.5), \\
(1.75,5.25),(3.5,5.25),(5.25,5.25)\end{array}$ & 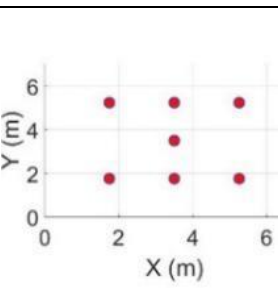 \\
\hline $\begin{array}{c}8 \text { Transmitters } \\
\text { coordinates: } \\
(1.4,1.75),(2.8,1.75), \\
(4.2,1.75),(5.6,1.75), \\
(1.4,5.25),(2.8,5.25), \\
(4.2,5.25),(5.6,5.25)\end{array}$ & 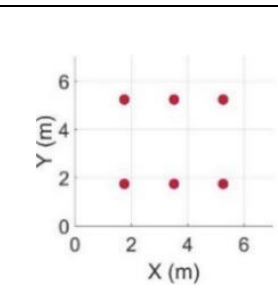 \\
\hline
\end{tabular}

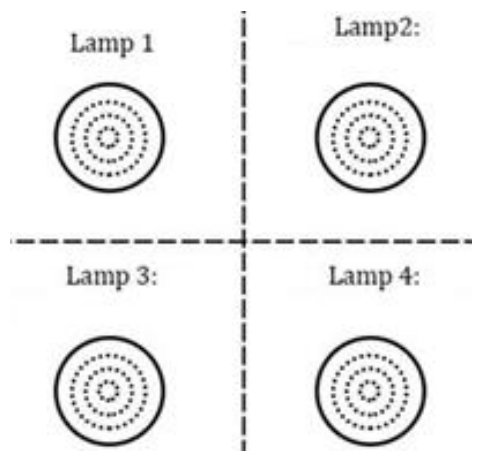

Fig. 5 An array of LEDs are commonly used to enhance the illumination efficiency.

Table 2 shows the coordinates and allocations of eight different LED transmitters for a $7 \times 7 \times 3 \mathrm{~m}$ room dimension. One can notice that whenever the number of LEDs is odd, a preference in terms of increasing the number of LEDs is given to the room center, where probably people are mostly residing most of the time. Further, it worth mentioning that an array of LEDs as shown in Fig. 5 is typically used to satisfy the illumination requirement, which is used in our simulation. Thus, each dot in the LEDs arrangement of Table 2 consists of an array of LEDs.

Table 3 depicts the illumination results for a room of dimension $7 \times 7 \times 3 \mathrm{~m}$ with different number of transmitters. From the results, it can be seen that even for a room of such large size, close to $100 \%$ illumination efficiency is attained which indeed satisfies the ISO standards for number of transmitters that 
equals to 6 or more. Furthermore, this result advocates the effectiveness of our proposed LEDs grid arrangement that is utilized in the room ceiling. Additionally, Table 4 verifies the above results by showing a grey colored figures, where areas that satisfy the ISO illumination standard are colored in light gray, while the ones that do not satisfy it are colored in dark gray. Yet again, it is clear that when the number of LEDs is increased to 6, illumination efficiency is satisfied in accordance with the ISO illumination standard. For further illustration, the table shows the contour and mesh diagrams for various light distributions. It is noteworthy that we have carried out the simulation experiments for different room sizes. From the results, we infer that for each room size, we require a minimum number of LEDs to satisfy the ISO illumination requirements, so that both the light illumination and data transmission efficiency can be jointly studied. This will aid us to decide on the minimum number of LEDs that will yield satisfactory light illumination thereby resulting in improved data transmission efficiency. Consequently, in the next section, the performance evaluation in terms of light illumination and data transmission efficiency is investigated for different light sources positions.

Table 3 Illumination results of different number of LEDs utilizing a grid arrangement for a room dimension of $7 \times 7 \times 3 \mathrm{~m}$

\begin{tabular}{|c|c|c|}
\hline $\begin{array}{c}\text { Number of LED } \\
\text { array transmitters }\end{array}$ & $\begin{array}{c}\text { Average luminance } \\
\text { of the room }\left(l_{x}\right)\end{array}$ & $\begin{array}{c}\text { Percentage of room average illumination } \\
\text { satisfying ISO standards (\%) }\end{array}$ \\
\hline 1 & 149.99 & 4.46 \\
\hline 2 & 272.34 & 38.94 \\
\hline 3 & 422.33 & 71.95 \\
\hline 4 & 495.01 & 97.61 \\
\hline 5 & 645.01 & 99.92 \\
\hline 6 & 767.35 & 100 \\
\hline 7 & 917.35 & 100 \\
\hline 8 & 1009.5 & 100 \\
\hline
\end{tabular}

\subsection{LEDs’ Data Transmission Performance Evaluation}

After studying the effect of changing the number of LEDs and their locations in a room light for illumination efficiency, it is important to study the performance of the data transmission ability, thus assuring that the LEDs can have acceptable performance for both light illumination and data transmission. In particular, several design parameters such the number of transmitters, receivers, and the room dimensions affect the data transmission performance. Hence, one of these parameters is kept fixed while the others are varied in our simulation, as will be discussed in the following scenarios. Table 5 shows the additional parameters used to simulate the VLC system. 
Table 4 The effect of changing the number of transmitters on the room light illumination intensity

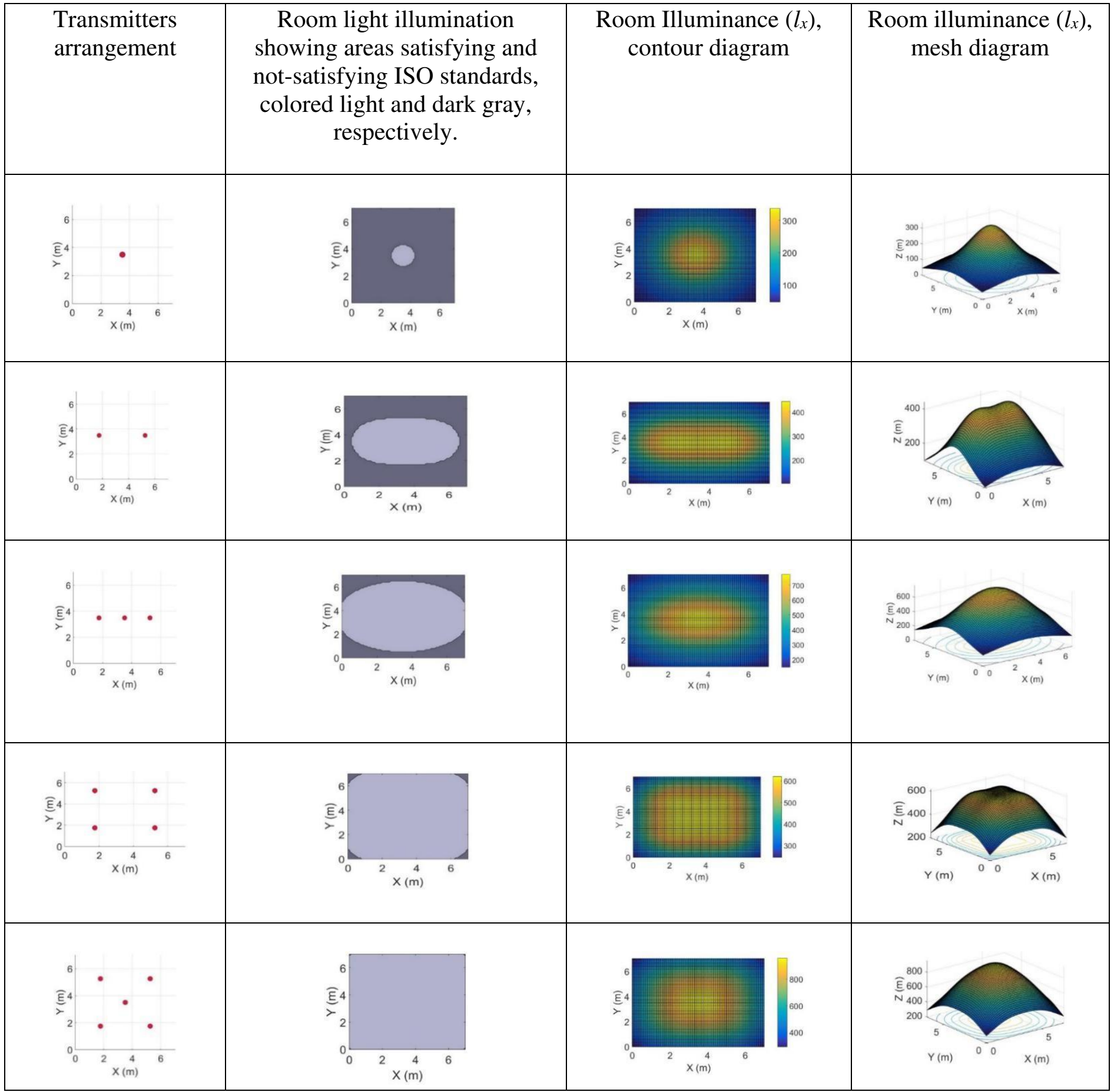




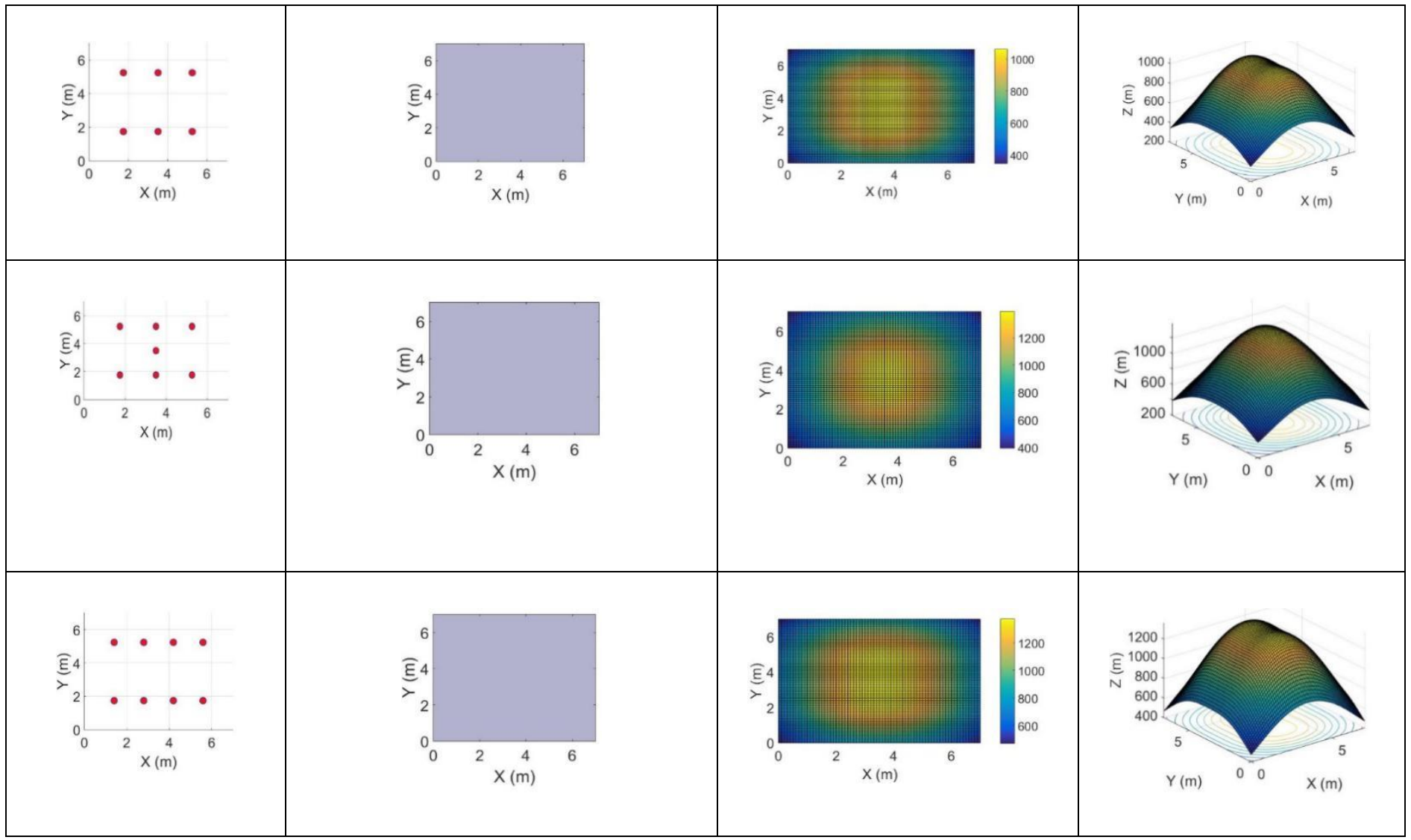

Table 5 Additional simulation parameters

\begin{tabular}{|c|c|}
\hline Transmitter mode number & 1 \\
\hline Emitting power & 1 \\
\hline Receiver area & $0.0001 \mathrm{~m}^{2}$ \\
\hline FOV & $85^{\circ}$ \\
\hline Reflections considered & 0.8 \\
\hline Reflection coefficient & 1,000 \\
\hline Number of rays used in the simulation & \\
\hline
\end{tabular}

\subsubsection{The effect of number of LED transmitters}

The first scenario investigates the effect of LED transmitters distributed in a grid setup as described before for different room sizes. The room is designed with multiple receivers that are deployed in grid formation with distance of $0.5 \mathrm{~m}$ between them. Figure $6(\mathrm{a}-\mathrm{d})$ shows the performance of the VLC communication system measured in terms of the attainable BER for different room sizes. As expected, it can 
be noticeable that the system performance is improved as the number of transmitter increases, especially when the SNR is low. However, at higher SNR, increasing the number of LEDs has small impact on improving the performance. One potential application scenario in this context can be transmitting the multimedia contents for the users who can tolerate small distortion in the multimedia content. As such for the case, increasing the number of LEDs is not going to result in a visible impact on the media content. Hence, the number of LEDs can be set to meet the ISO lightening standard ( 6 for the case of $7 \times 7 \times 3 \mathrm{~m}$ room dimensions).

\subsubsection{The effect of the room dimension}

In order to have a closer look at the effect of changing the room dimension, here, for each experiment, the simulation setup that was used in the earlier setting is adopted. However, in this experiment the number of transmitters and receivers is fixed while the room dimension keeps changing. Besides, note that we have employed the same receiver configurations used in the previous scenario. The system performance is measured for different SNR values. As depicted in Figs. 7(a-d) and Figs. $8(\mathrm{e}-\mathrm{h})$, regardless of the number of transmitters, the smaller room dimension has always resulted in better performance than the larger dimensions. This is due to the fact that the light path loss will be lower and the energy level will be higher in case of smaller room dimensions. Further, the effect of multi-path will be insignificant in the case of smaller room dimensions, which will not be the case in larger room dimension. Furthermore, when the SNR is low $(<4 \mathrm{~dB})$ and the number of transmitters is $\leq 5$, the system performance for all room dimensions is almost the same. However, increasing the number of transmitters more than 5 have a noticeable impact especially at low SNR values (from 4 to $6 \mathrm{~dB}$ ). At higher SNR values (i.e., >8), the performance enhancement resulted from increasing the number of transmitters $(>3)$ is more significant. This is apparent particularly for small room dimensions $(4 \times 4 \times 3 \mathrm{~m}$ and $5 \times 5 \times 3 \mathrm{~m})$ than the cases of having larger room dimension $(6 \times 6 \times 3$ $\mathrm{m}$ and $7 \times 7 \times 3 \mathrm{~m})$ and higher number of transmitters (i.e., $>3$ ).

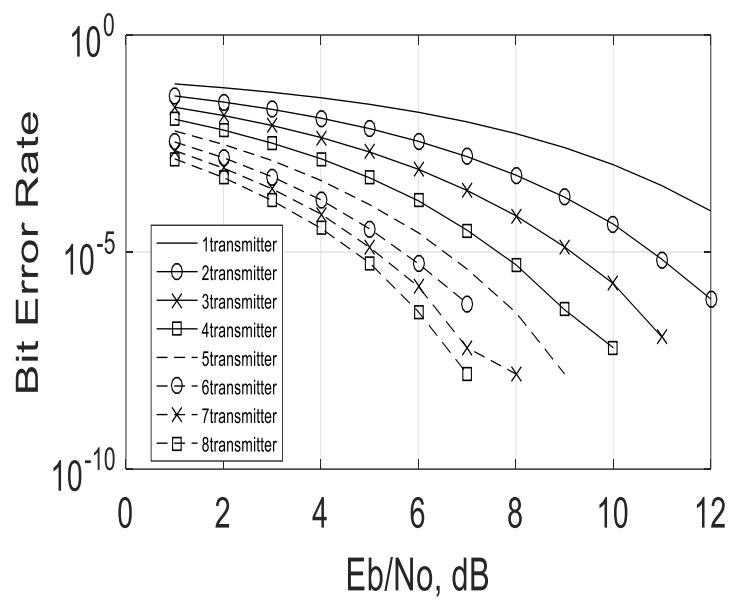

(a)

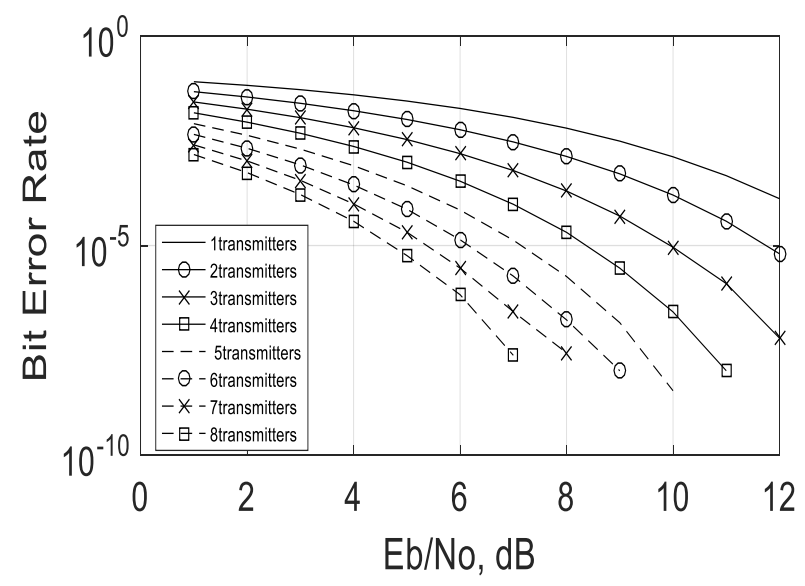

(b) 


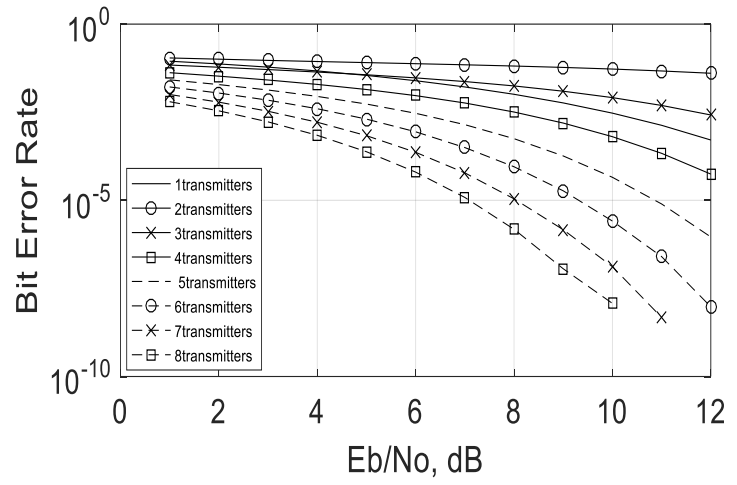

(c)

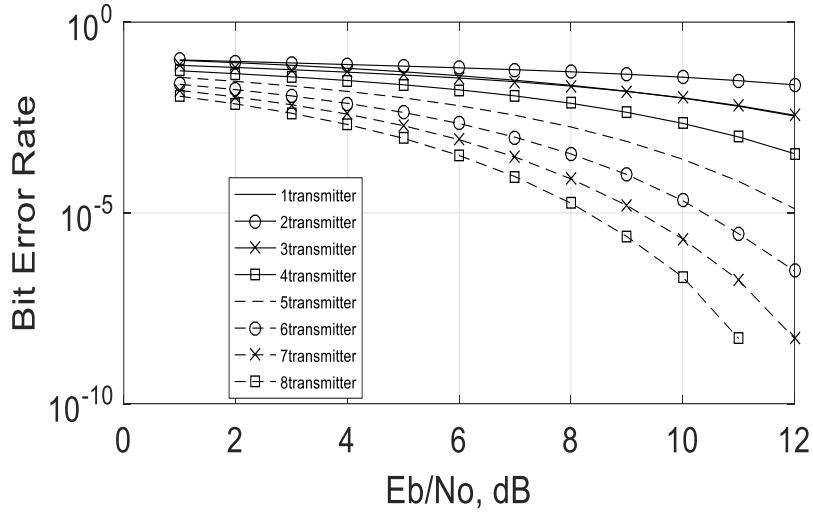

(d)

Fig. 6 The VLC system performance for different number of transmitters and receivers and different room dimensions: (a) $4 \times 4 \times 3 \mathrm{~m}$, (b) $5 \times 5 \times 3 \mathrm{~m}$, (c) $6 \times 6 \times 3 \mathrm{~m}$, and (d) $7 \times 7 \times 3 \mathrm{~m}$.

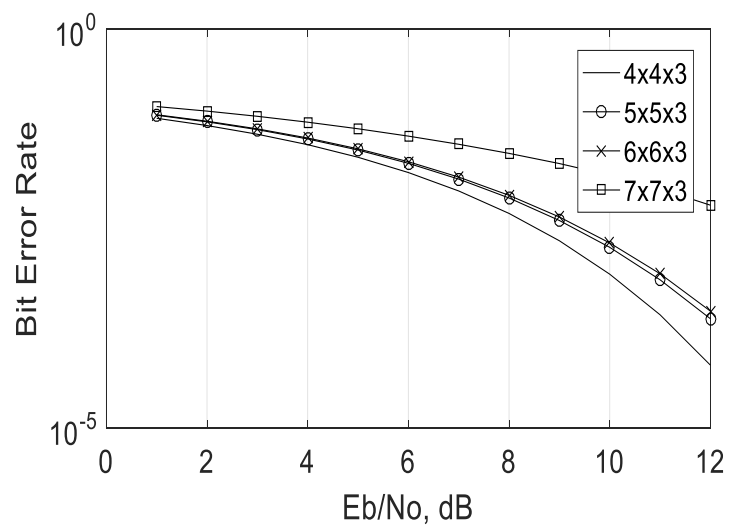

(a)

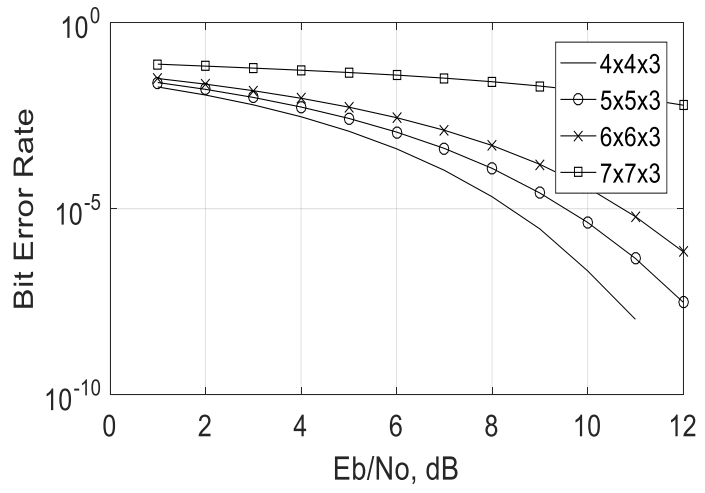

(c)

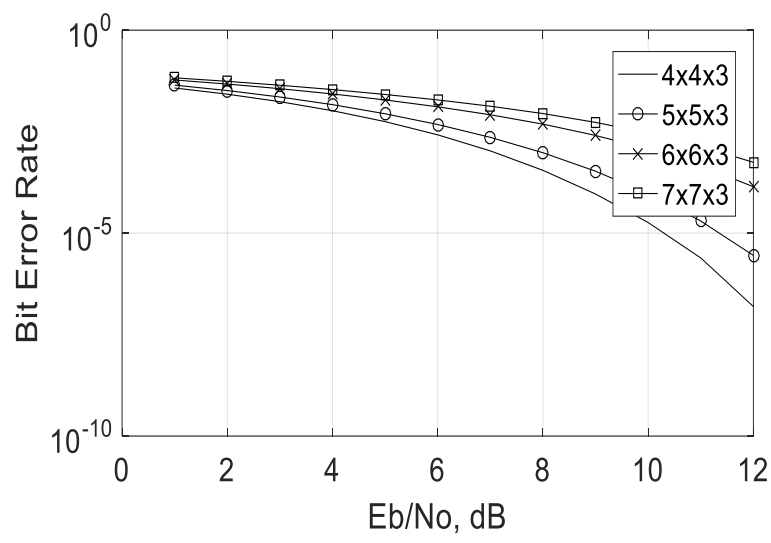

(b)

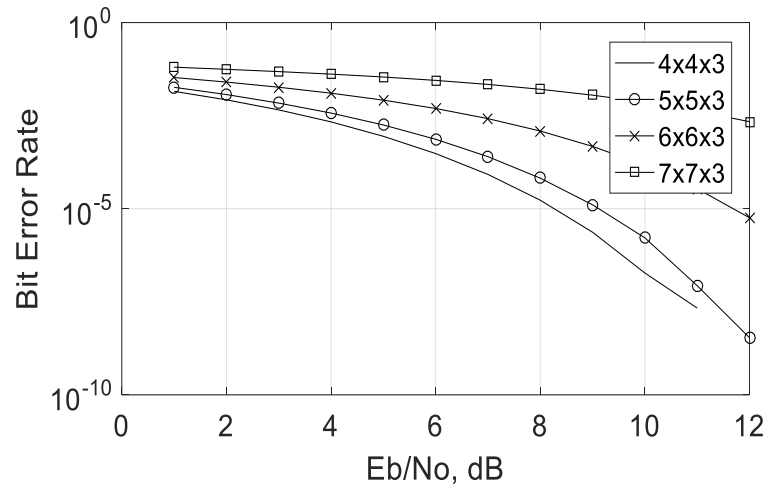

(d)

Fig. 7 The VLC system performance while varying the room dimensions and changing the number of transmitters: (a) 1 (b) 2 (c) 3 (d) 4 transmitters. 


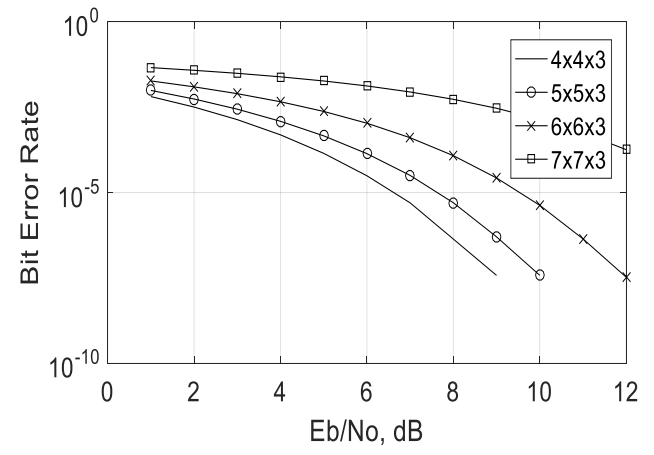

(a)

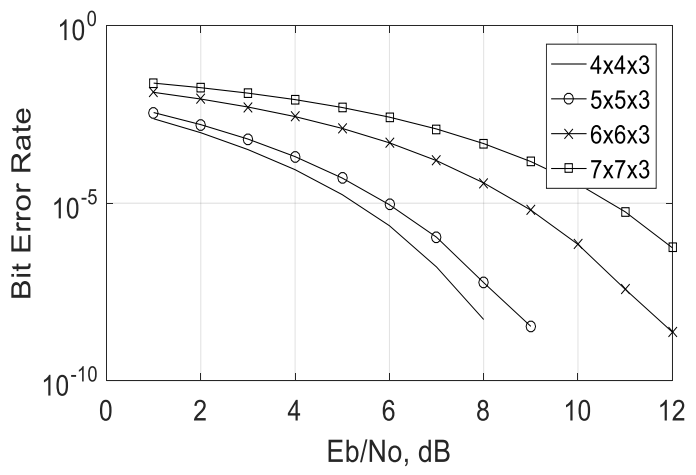

(c)

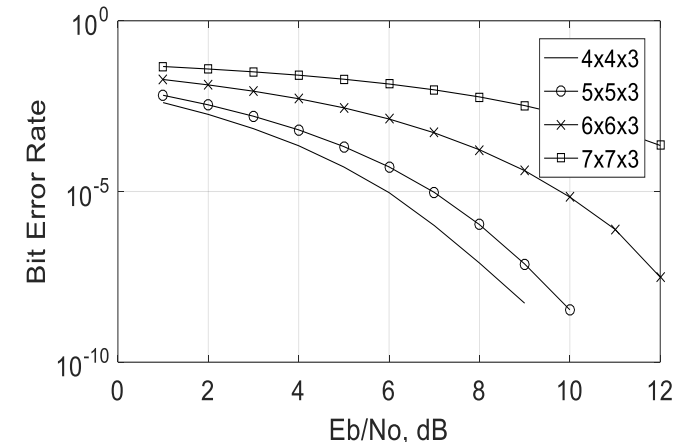

(b)

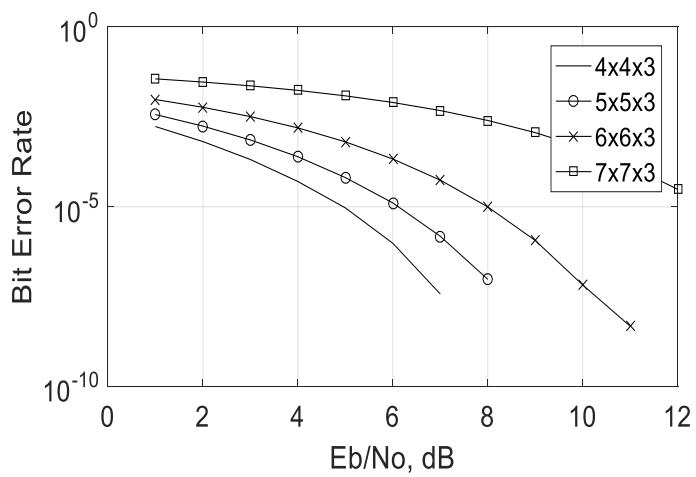

(d)

Fig. 8 The VLC system performance while varying the room dimensions and changing the number of transmitters: (a) 5 (b) 6 (c) 7 (d) 8 transmitters.

\subsection{Video transmission over VLC use case}

Multimedia transmission is one potential application for using VLC where it can be used to transmit video and images containing promotions, ads, and coupons, to potential customers while shopping and utilizing the shop lights. For example, a customer can position his/her smartphone under a product display that is running a special program capable of receiving and decoding the light signal and interpret it to a coupon that can be used to get a discount on the displayed product. In this section, for the video transmission we have selected a normal video in AVI format for transmission purpose. It has 300 frames in the ratio 4:3. The video obtained is then read as binary in our code. It is then transmitted through the designed channel. The received video after passing through the channel is obtained as binary and then written into AVI format video. Now, for every trial and case, we have a source video and the received video.

Next, we need to compare the received videos to check its quality. Therefore, the source video and the received video are to be compared. There are two techniques for comparing the quality of the video: peak signal-to-noise ratio (PSNR) and structural similarity (SSIM) techniques. We have used the SSIM technique for the quality evaluation, as it is more apt for the video file. SSIM is known as structural similarity index, a metric known for evaluation of image and video quality. SSIM is used for the measurement of similarity between two images. In SSIM, a distorted image is compared with the original uncompressed image as the reference. SSIM is an improved technique to the traditional PSNR technique.

In our code, the source video before transmission through the channel and the received video after passing through the channel are compared using the SSIM technique. In this code, both the videos are separated into various frames. Each corresponding frame is compared with each other and their SSIM value 
is calculated. Finally, the average of the entire SSIM value is taken to get the SSIM index for the videos compared. This process takes place for all the videos received. The SSIM index values vary from 0 to 1. Higher the value less is the distortion in the received video and better is the quality. Lower the value, it means that there is more distortion in the received video and hence, the quality is low. The value 1 represents the least distorted and 0 being the most distorted. Therefore, by the process of video transmission through the channel, we can check the efficiency of our channel and the effects related to different number of transmitters, different room dimensions, and different number of samples per second.

In order to understand how different video parameters affect the video quality, an objective video quality evaluation metric is used. The SSIM) [27] index is a method for measuring the similarity between two images. SSIM is designed to improve on traditional methods like (PSNR) and mean-squared error (MSE) [28]. The SSIM metric is calculated on various windows of an image. The measure between two windows $x$ and $y$ of common size $N \times N$ is described by Equation (10).

where:

$$
\operatorname{SSIM}(x, y)=\frac{\left(2 \mu_{x} \mu_{y}+c_{1}\right)\left(2 \sigma_{x y}+c_{2}\right)}{\left(\mu_{x}^{2}+\mu_{y}^{2}+c_{1}\right)\left(\sigma_{x}^{2}+\sigma_{y}^{2}+c_{2}\right)}(10),
$$

- $\mu_{x}{ }^{2}$ : the average pixel values of a reference image (x )

- $\mu_{y}{ }^{2}$ : the average pixel values of a reference image (y )

- $\sigma_{x}^{2}:$ the variance of $x$

- $\sigma_{y}{ }^{2}:$ the variance of $y$

- $\sigma_{x y}$ : the covariance of $x$ and $y$

- $c_{1}=\left(k_{1} L\right)^{2}, c_{2}=\left(k_{2} L\right)^{2}, L=2^{\# \text { bitsperpixel }}-1$ and $k_{1}=0.01, k_{2}=0.03$

The video quality is estimated by calculating the average of the SSIM values of the video images.

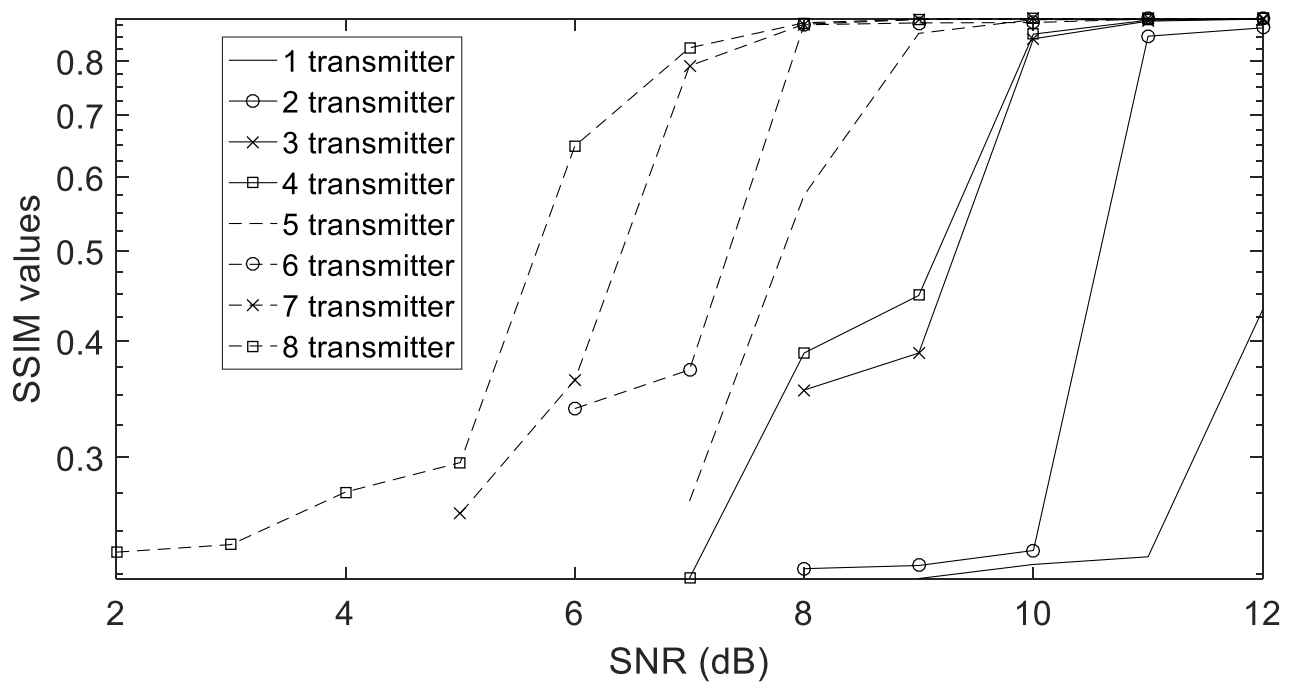

Fig. 9 SSIM values for video transmission over VLC channel for different SNR values and number of transmitters 
As seen from Fig. 9, the SNR (dB) and SSIM values are plotted by varying the number of transmitters. It is seen that, even though all the curves reach the SSIM value of 1 at one point, it takes higher SNR values for lesser number of transmitters to reach high quality. Hence, it is clear that for higher number of transmitters, better video quality can be achieved at small SNR values. In addition, it is obvious that more the number of transmitters better is the received video quality, which shows the efficiency of the proposed VLC system for multimedia transmission.

\section{CONCLUSION AND FUTURE WORK}

In this paper, a simulation framework to study the LEDs lighting functionality and data transmission efficiency measured in terms of light intensity based on ISO standard and BER, respectively, for a VLC system, has been proposed. The outcome of our study can be used as a guideline to design a VLC system such that a suitable number of LEDs can be used to satisfy the lighting and data rates requirements for application under consideration. As a future course of work, we are working in evaluating the optimum number of transmitters and receivers configurations that will ensure the transmission quality for different multimedia signals for different room dimensions.

\section{DECLARATION}

Funding: This work has been funded in part by the German Jordanian University seed fund ID SEEIT $02 / 2018$

Conflicts of interest/Competing interests: Not applicable

Availability of data and material (data transparency): Not applicable

Code availability (software application or custom code): Not applicable

Authors' contributions: Ala' F. Khalifeh: Paper idea, writing, simulation coding and setup, Karthikeyan

Alakappan: Paper writing, simulation coding and results' analysis. Barath Kumar: Paper writing,

simulation coding and results' analysis, Jayanth Prabakaran: Paper writing, simulation coding and results' analysis ,Prabagarane Nagaradjane: Paper writing.

\section{REFERENCES}

[1]L. Hanzo, H. Haas, S. Imre, D. O’Brien, M. Rupp, and L. Gyongyosi, "Wireless Myths, Realities, and Futures: From 3G/4G to Optical and Quantum Wireless," Proceedings of the IEEE, vol. 100, pp. 1853-1888, 2012.

[2]R. Zhang, J. Wang, Z. Wang, Z. Xu, C. Zhao, and L. Hanzo, "Visible light communications in heterogeneous networks: Paving the way for user-centric design," IEEE Wireless Communications, vol. 22, no. 2, pp. 8-16, 2015.

[3]J. M. Kahn and J. R. Barry, "Wireless infrared communications," Proceedings of the IEEE, vol. 85, no. 2, pp. 265-298, 1997.

[4]C. Tsai and Z. Xu, "Line-of-Sight Visible Light Communications WithInGaN-Based Resonant Cavity LEDs," IEEE Photonics Technology Letters, vol. 25, no. 18, pp. 1793-1796, 2013.

[5]T. Komine and M. Nakagawa, "Integrated system of white LED visible-light communication and powerline communication," in The 13th IEEE International Symposium on Personal, Indoor and Mobile Radio Communications, 2002, vol. 4, pp. 1762-1766 vol.4.

[6]A. Jovicic, J. Li, and T. Richardson, "Visible light communication: opportunities, challenges and the path to market," IEEE Communications Magazine, vol. 51, no. 12, pp. 26-32, 2013.

[7]Khalifeh, Ala', F., Farahneh, H., Mekhiel, C., and Fernando, X., "Visible Light Communication Numerous Applications," Encyclopedia of Information Science and Technology, IGI Global, vol. Fourth 
Edition, pp. 6672-6683, 2018.

[8]R. CHENG and X. YAN, "Indoor multisource channel characteristic for visible light communication,"

The Journal of China Universities of Posts and Telecommunications, vol. 20, pp. 106-111, 2013.

[9]T. Komine and M. Nakagawa, "Fundamental analysis for visible-light communication system using LED lights," IEEE Transactions on Consumer Electronics, vol. 50, no. 1, pp. 100-107, 2004.

[10]Z. Yu, R. J. Baxley, and G. T. Zhou, "Multi-user MISO broadcasting for indoor visible light communication," in 2013 IEEE International Conference on Acoustics, Speech and Signal Processing, 2013, pp. 4849-4853.

[11]H. Le Minh et al., "Short-range Visible Light Communications," In Proceedings of the Wireless World Research Forum, Chennai, India,2007.

[12]H. Q. Nguyen et al., "A MATLAB-based simulation program for indoor visible light communication system," in 2010 7th International Symposium on Communication Systems, Networks Digital Signal Processing (CSNDSP 2010), 2010, pp. 537-541.

[13]Yu Yang, Xiongbin Chen, Lin Zhu, Bo Liu, and Hongda Chen, "Design of indoor wireless communication system using LEDs," in 2009 Asia Communications and Photonics conference and Exhibition (ACP), 2009, vol. 2009-Supplement, pp. 1-8.

[14]Z. Wu, J. Chau, and T. Little, "Modeling and designing of a new indoor free space visible light communication system," in 2011 16th European Conference on Networks and Optical Communications, 2011, pp. 72-75.

[15]F. Ahmed, S. Ali, and M. Jawaid, "A Review of Modulation Schemes for Visible Light Communication, International Journal Of Computer Science And Network Security, vol. 18, pp. 117-125,2018.

[16]Y. Zhu, W. Liang, J. Zhang, and Y. Zhang, "Space-Collaborative Constellation Designs for MIMO Indoor Visible Light Communications," IEEE Photonics Technology Letters, vol. 27, no. 15, pp. 1667-1670, 2015.

[17]L. Matheus, A. Borges, L. Vieira, M. Vieira, and O. Gnawali, "Visible Light Communication: Concepts, Applications and Challenges,” IEEE Communications Surveys \& Tutorials, vol. PP, pp. 1-1, 2019

[18]A. F. Khalifeh, N. AlFasfous, R. Theodory, S. Giha, and K. A. Darabkh, "An experimental evaluation and prototyping for visible light communication," Computers \& Electrical Engineering, vol. 72, pp. 248265,2018

[19]A. F. Khalifeh, N. AlFasfous, R. Theodory, and S. Giha, "An experimental evaluation of visible light communication utilizing telecommunications instructional modelling system," in 2017 International Conference on Wireless Communications, Signal Processing and Networking (WiSPNET), 2017, pp. 18131817.

[20]A. Singh, A. Srivastava, V. A. Bohara, and G. S. V. R. K. Rao, "Performance of Indoor Visible Light Communication System Under Random Placement of LEDs," in 2019 21st International Conference on Transparent Optical Networks (ICTON), 2019, pp. 1-5.

[21]H. Farahneh, A. Khalifeh, and X. Fernando, "An outdoor multi path channel model for vehicular visible light communication systems," in 2016 Photonics North (PN), 2016, pp. 1-1.

[22]H. Farahneh, C. Mekhiel, A. Khalifeh, W. Farjow, and X. Fernando, "Shadowing effects on visible light communication channels," in 2016 IEEE Canadian Conference on Electrical and Computer Engineering (CCECE), 2016, pp. 1-5.

[23]A. F. Khalifeh, N. AlFasfous, R. Theodory, S. Giha, and K. A. Darabkh, "On the Effect of Light Emitting Diodes Positions on the Performance of an Indoor Visible Light Communication System," in 2019 IEEE Conference of Russian Young Researchers in Electrical and Electronic Engineering (EIConRus), 2019,

pp. 10-14.

[24]Marvin K. Simon and Mohamed-Slim Alouini, Digital Communication over Fading Channels, 2 nd ed. 
Wiley-IEEE Press, 2005.

[25]M. I. S. Chowdhury, W. Zhang, and M. Kavehrad, "Combined Deterministic and Modified Monte Carlo Method for Calculating Impulse Responses of Indoor Optical Wireless Channels," Journal of Lightwave Technology, vol. 32, no. 18, pp. 3132-3148, 2014.

[26]Y. Qiu, H.-H. Chen, and W.-X. Meng, "Channel modeling for visible light communications-a survey," Wireless Communications and Mobile Computing, vol. 16, pp. 2016-2034, 2016

[27] Z. Wang, A. C. Bovik, H. R. Sheikh and E. P. Simoncelli, "Image quality assessment: From error visibility to structural similarity," IEEE Transactions on Image Processing, vol. 13, no. 4, pp. 600-612, Apr. 2004.

[28] A. Khalifeh, M. Al-Taee, and A. Murshed, "Network-status aware quality adaptation algorithm for improving real-time video streaming over the internet", Multimed Tools Appl (2016). 
Figures

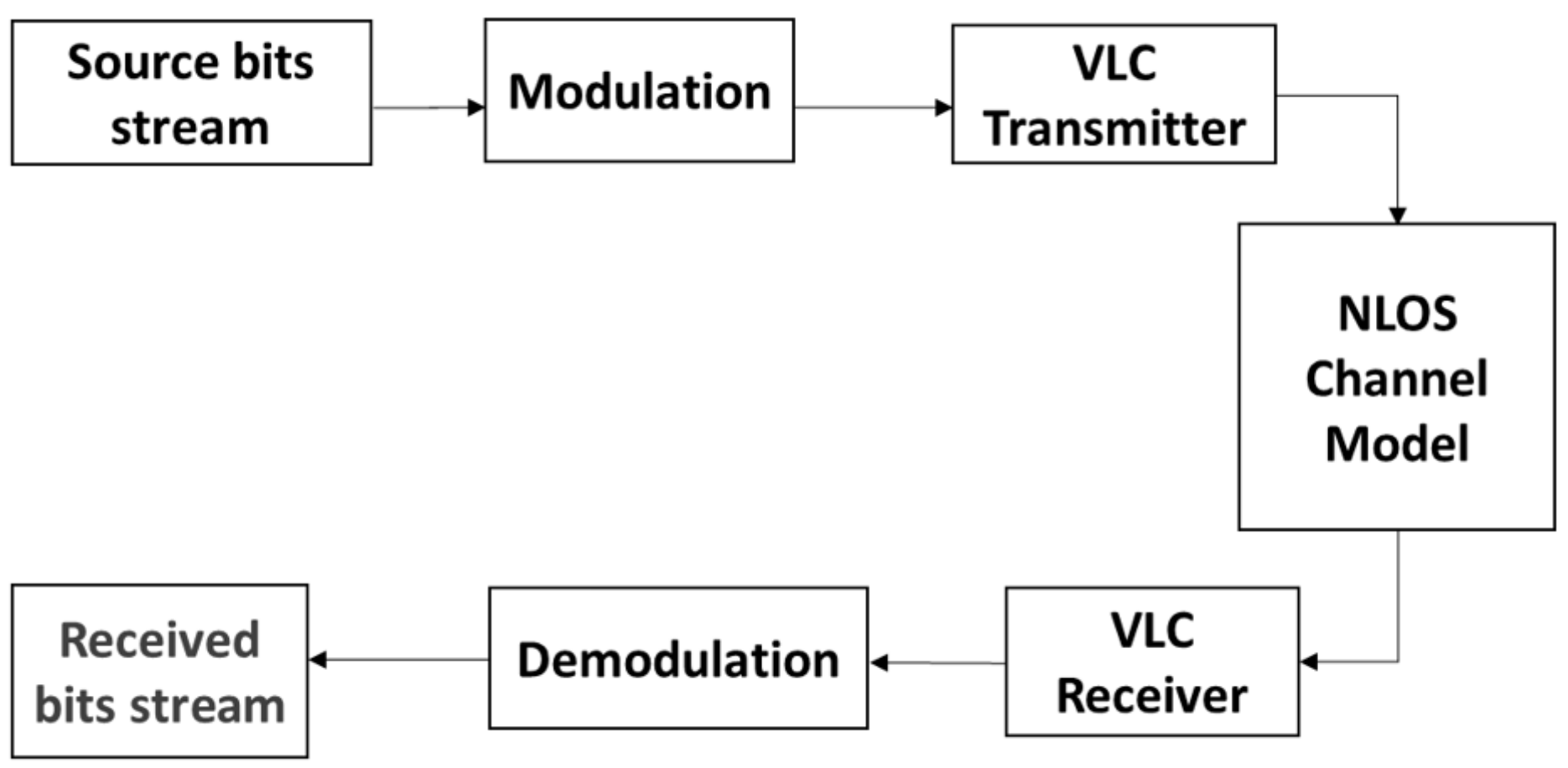

Figure 1

A block diagram of a VLC system. 


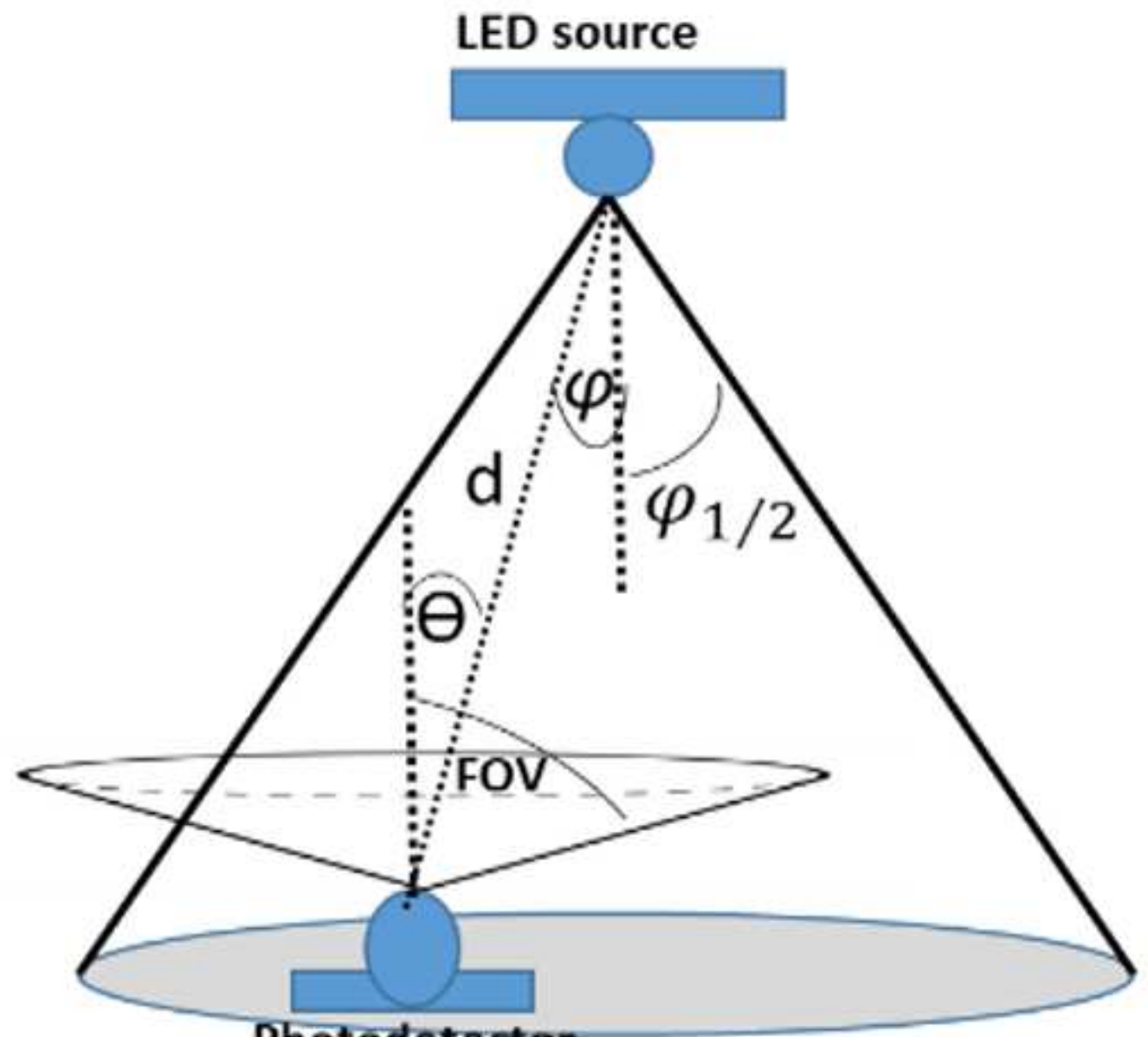

Photodetector

Figure 2

The main parameters used in characterizing an LED source and a photo-diode receiver [16]. 


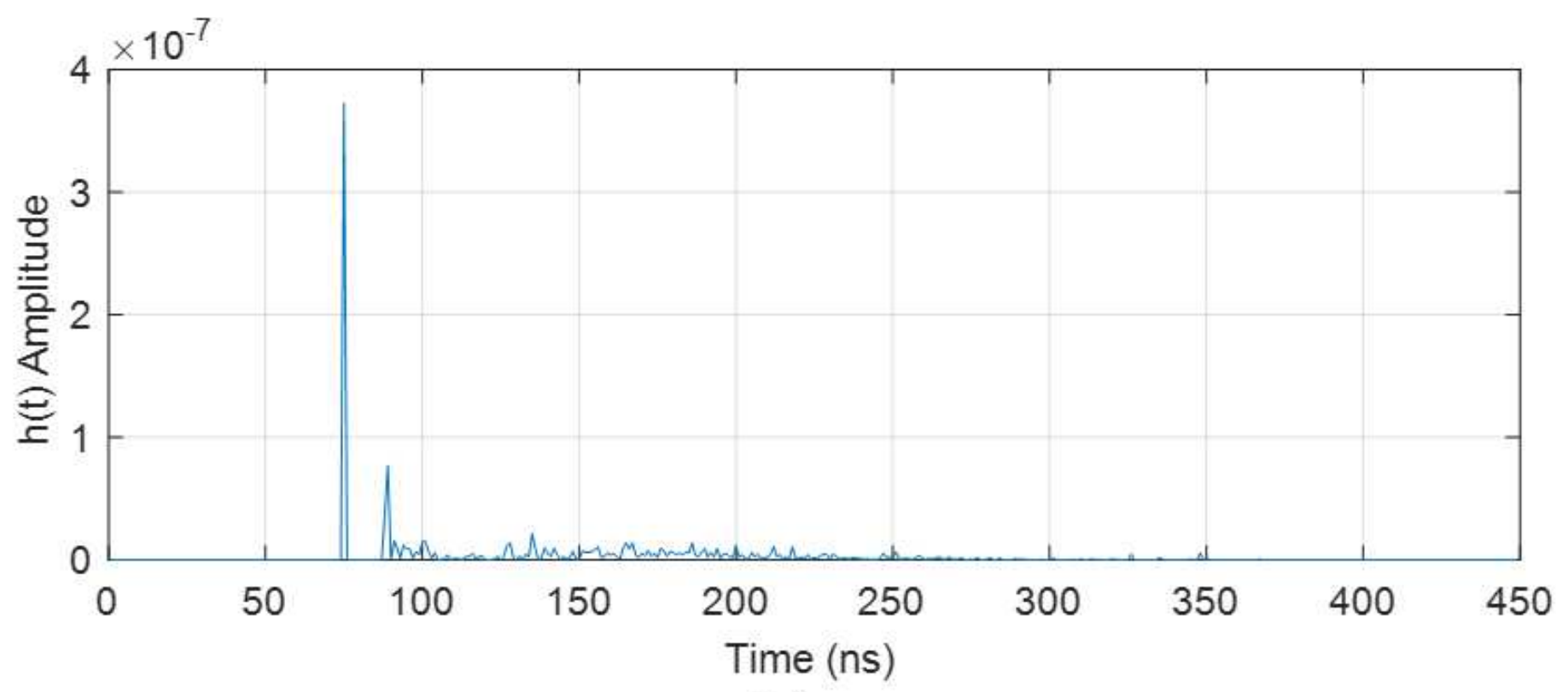

(a)

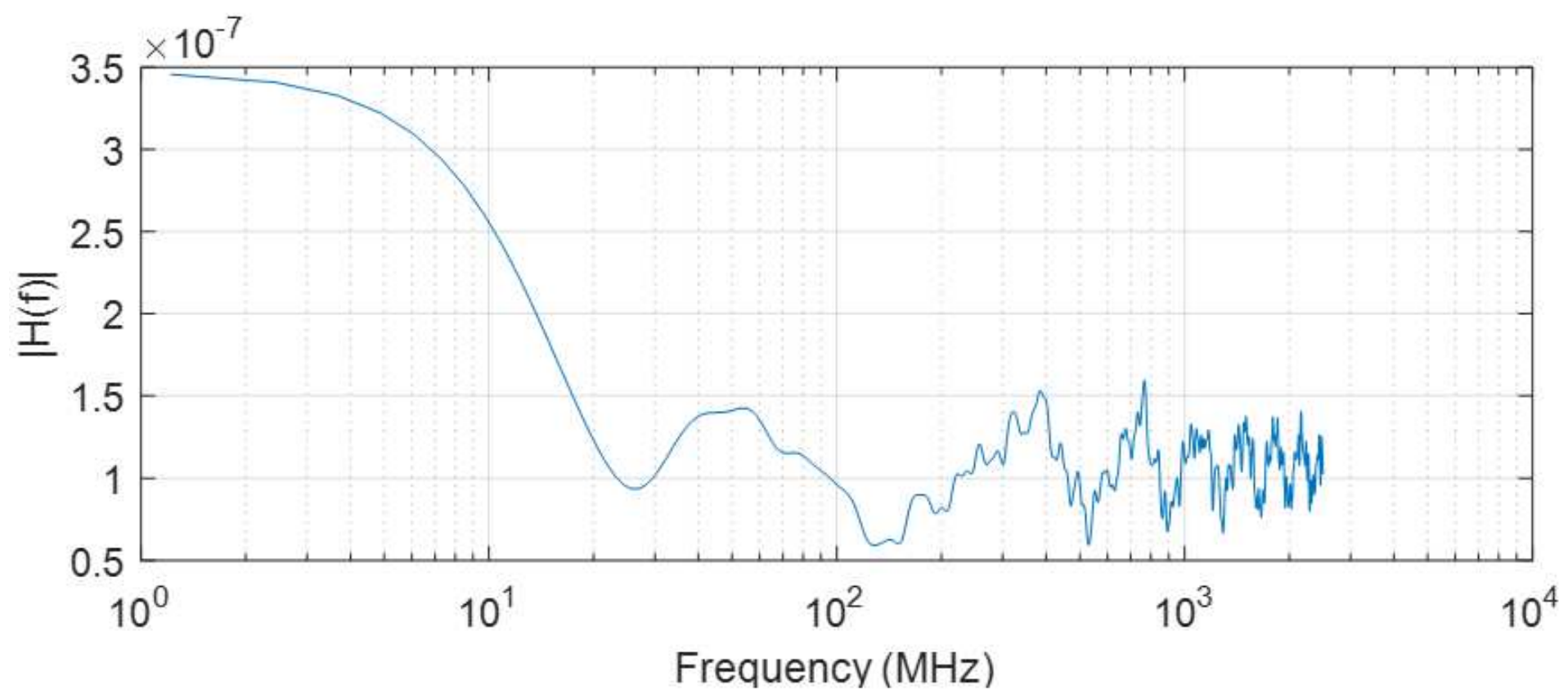

(b)

\section{Figure 3}

A NLOS impulse response in (a) time and (b) frequency domains for an indoor room of a single transmitter and receiver located at $[3.5,3.5,3],[0.5,1,3] \mathrm{m}$, respectively, in a room dimension of $[7,7,3] \mathrm{m}$. 


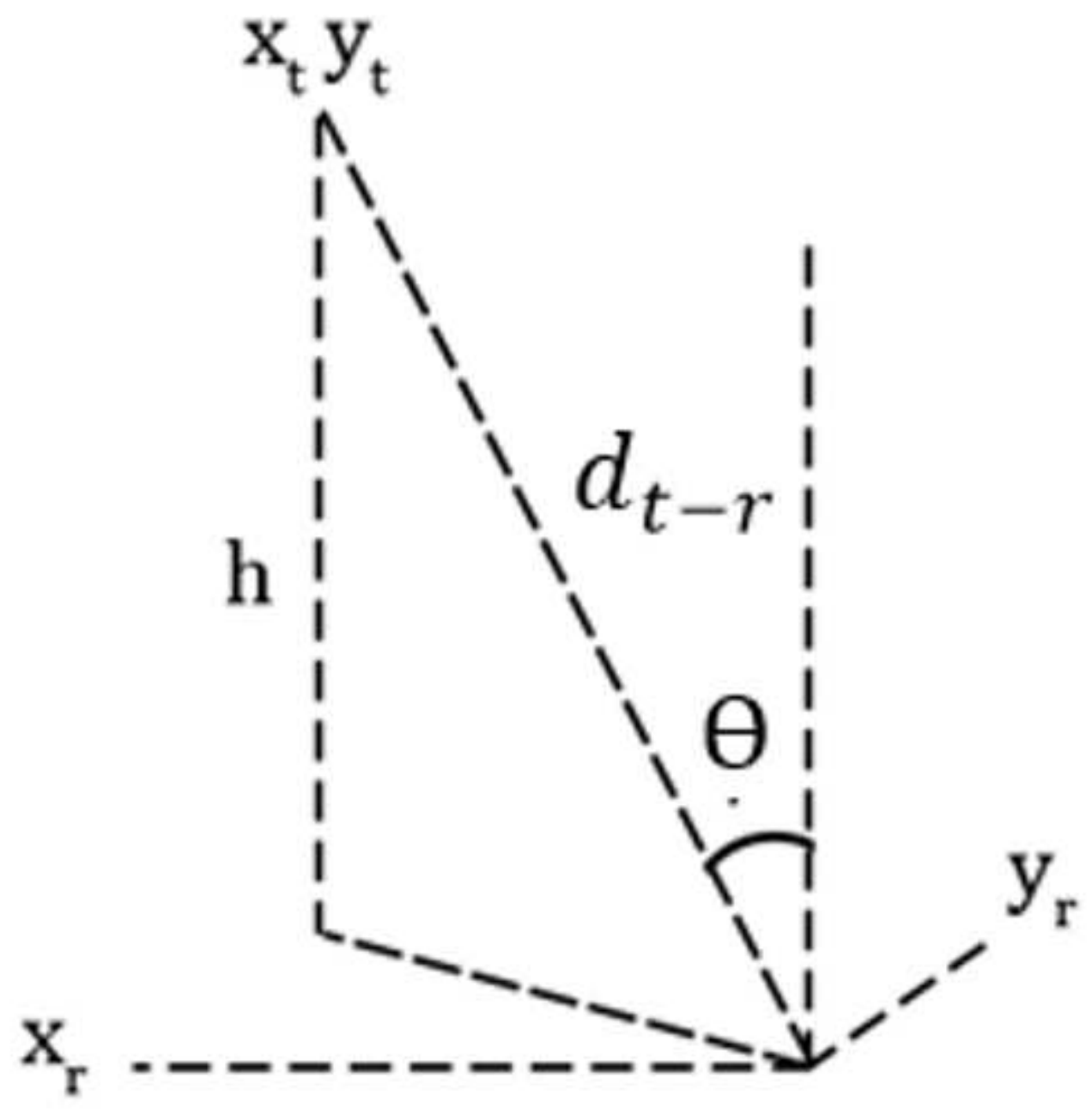

Figure 4

Assuming a fixed transmitter at $x t, y t$, the distance between that transmitter and any point on the receiver $\mathrm{xr}$, yr mesh-grid can be calculated using Pythagoras' theorem.

\section{Figure 5}

An array of LEDs are commonly used to enhance the illumination efficiency. 


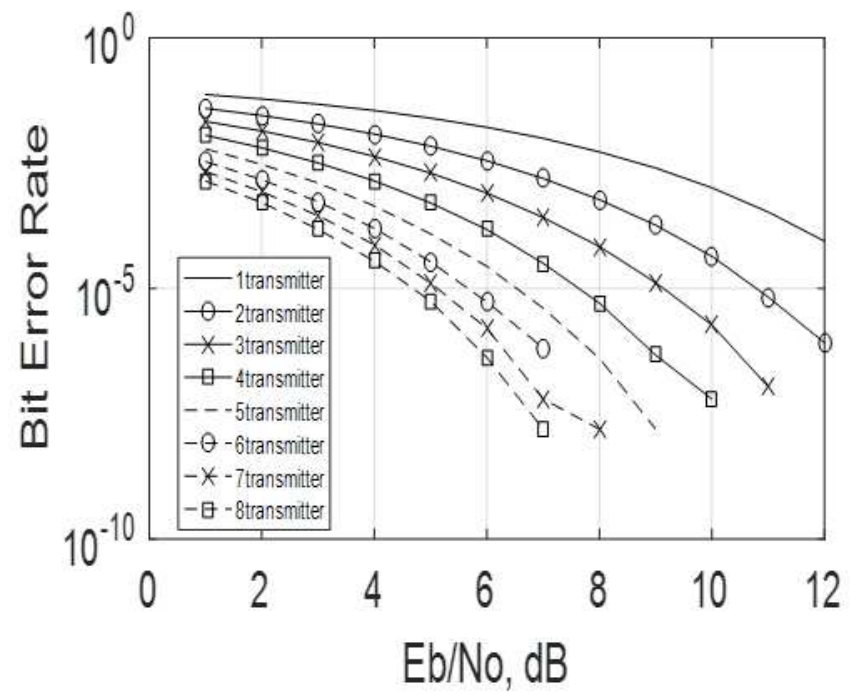

(a)

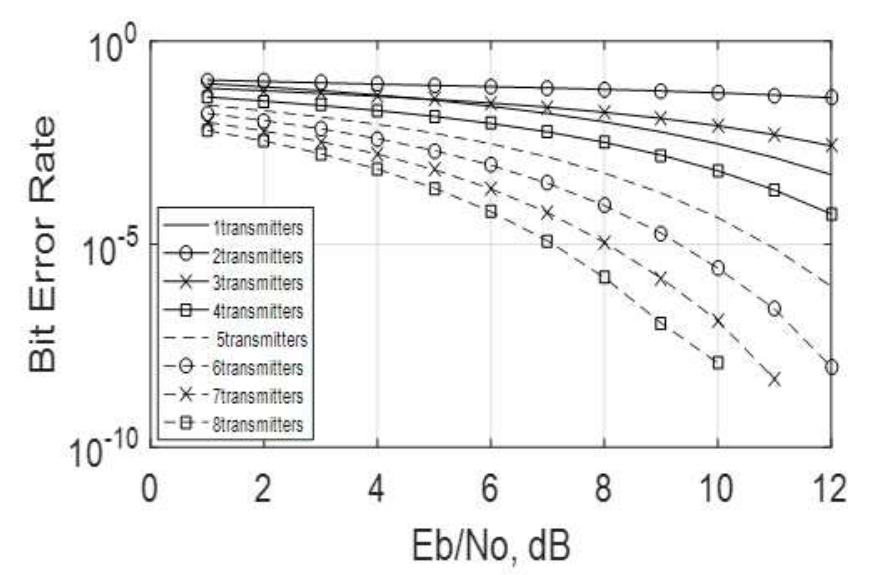

(c)

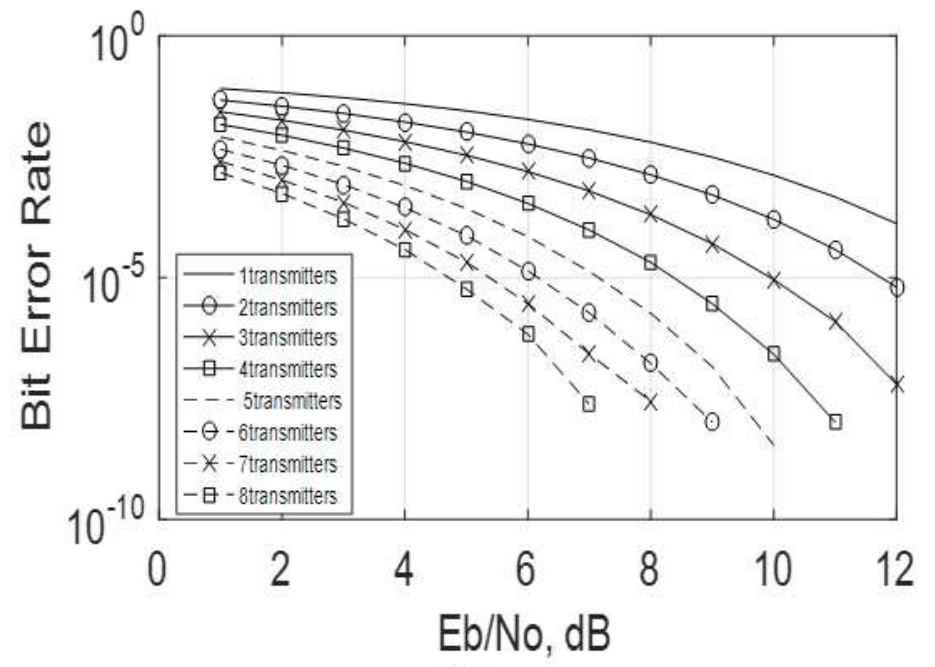

(b)

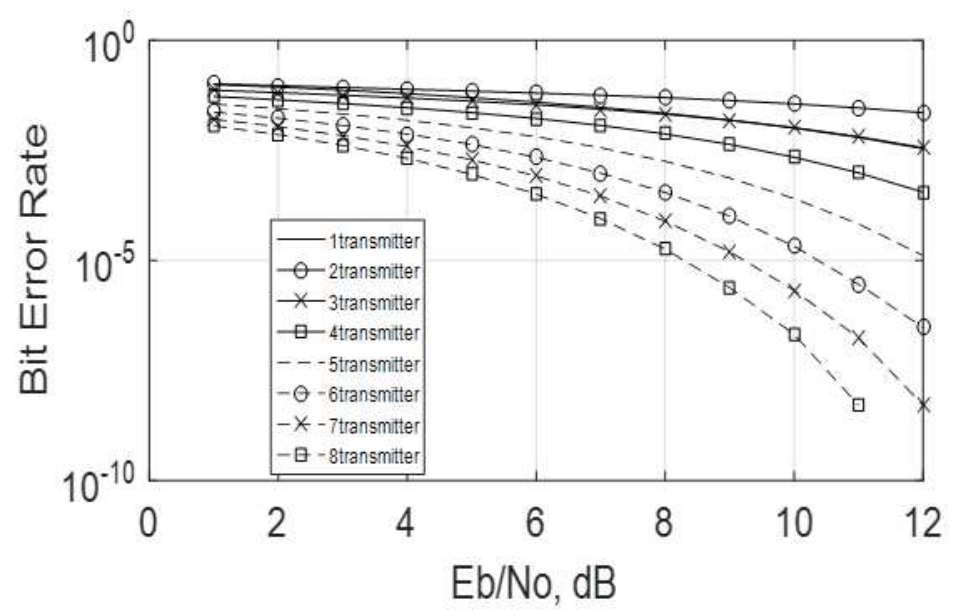

(d)

\section{Figure 6}

The VLC system performance for different number of transmitters and receivers and different room dimensions: (a) $4 \times 4 \times 3 \mathrm{~m}$, (b) $5 \times 5 \times 3 \mathrm{~m}$, (c) $6 \times 6 \times 3 \mathrm{~m}$, and (d) $7 \times 7 \times 3 \mathrm{~m}$. 


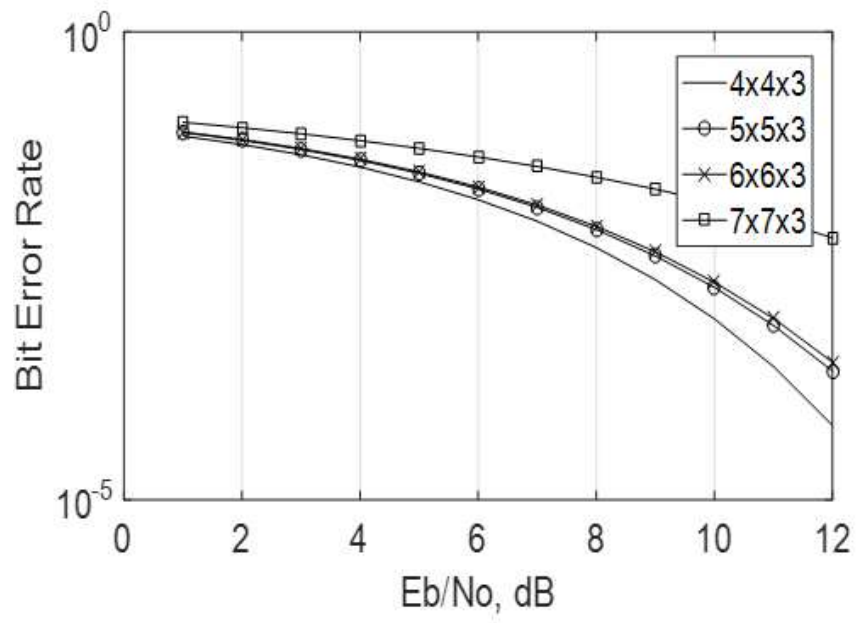

(a)

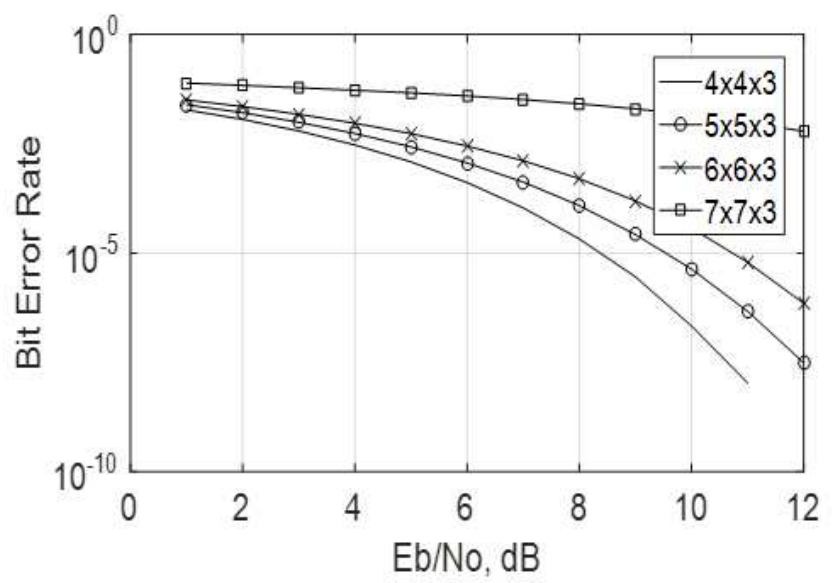

(c)

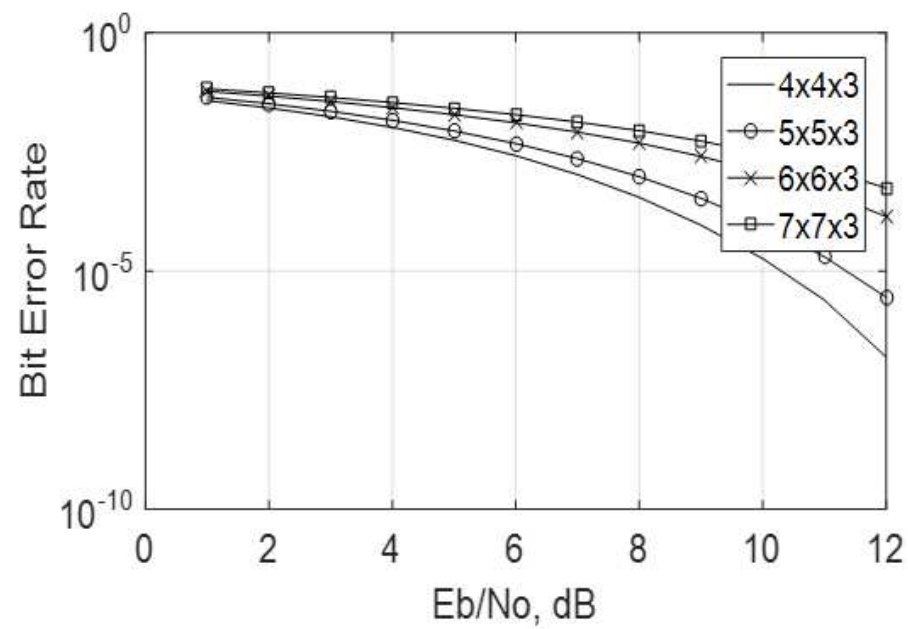

(b)

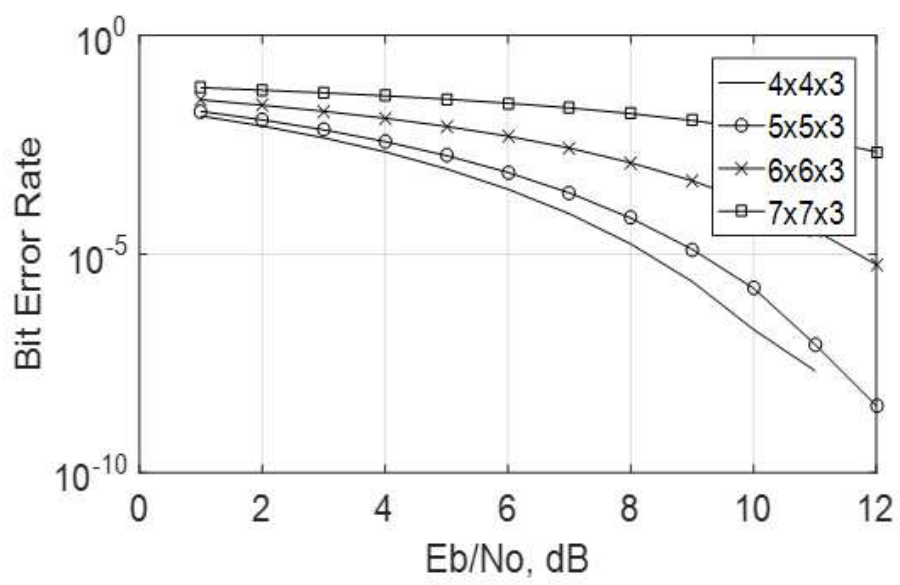

(d)

\section{Figure 7}

The VLC system performance while varying the room dimensions and changing the number of transmitters: (a) 1 (b) 2 (c) 3 (d) 4 transmitters. 


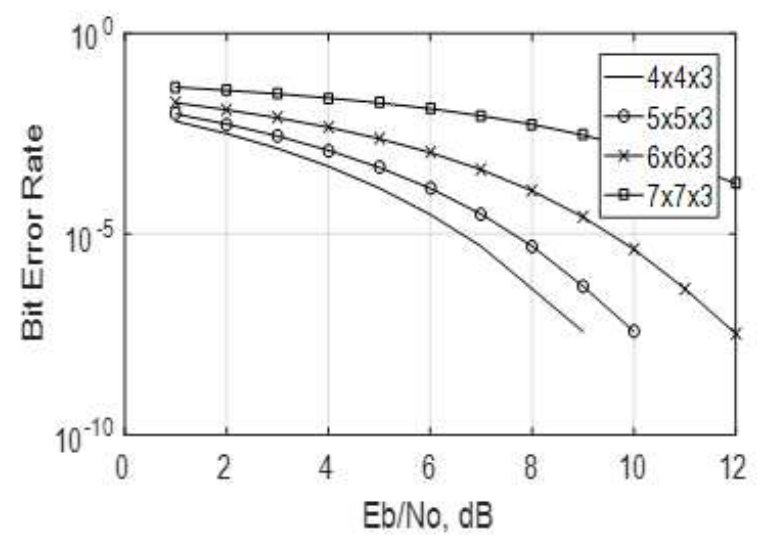

(a)

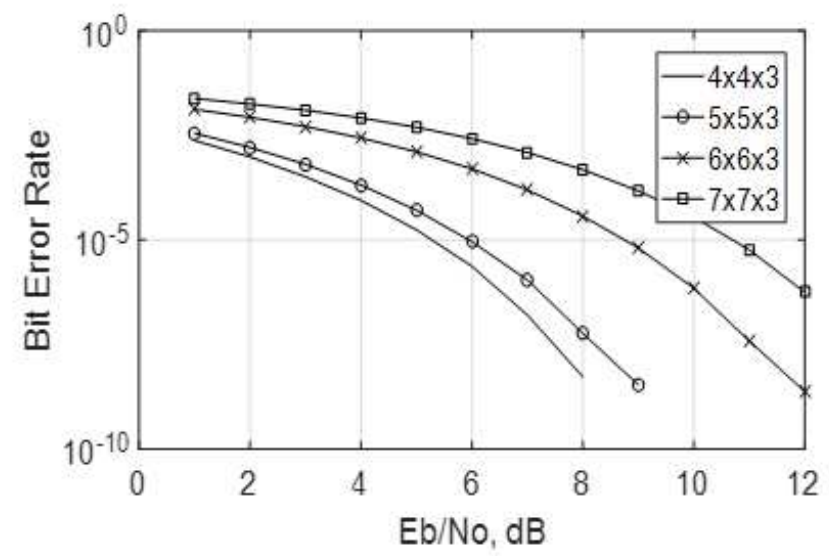

(c)

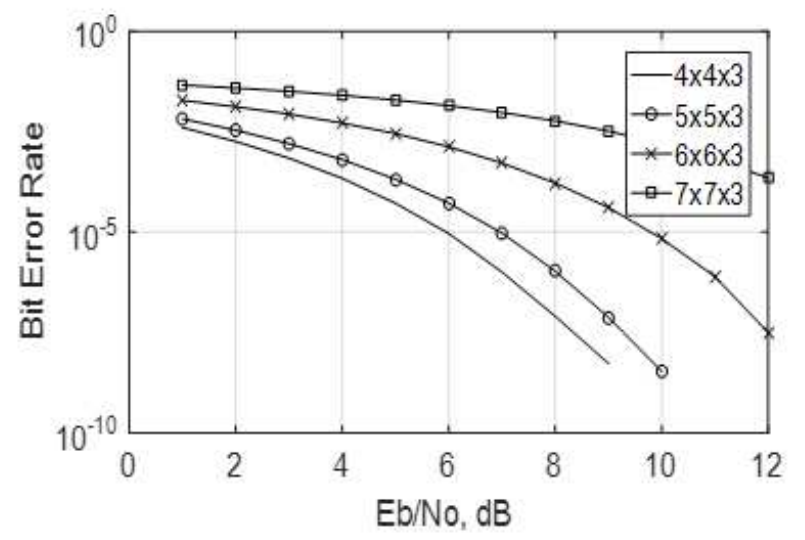

(b)

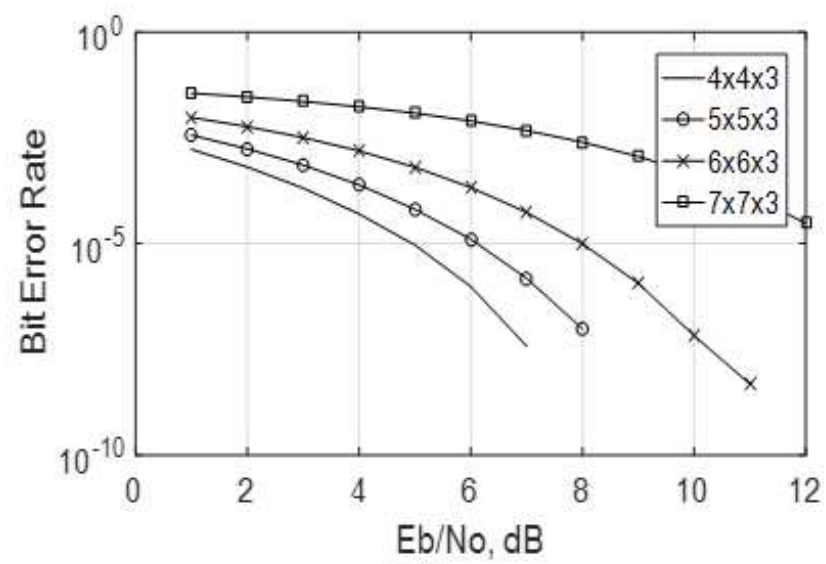

(d)

\section{Figure 8}

The VLC system performance while varying the room dimensions and changing the number of transmitters: (a) 5 (b) 6 (c) 7 (d) 8 transmitters. 


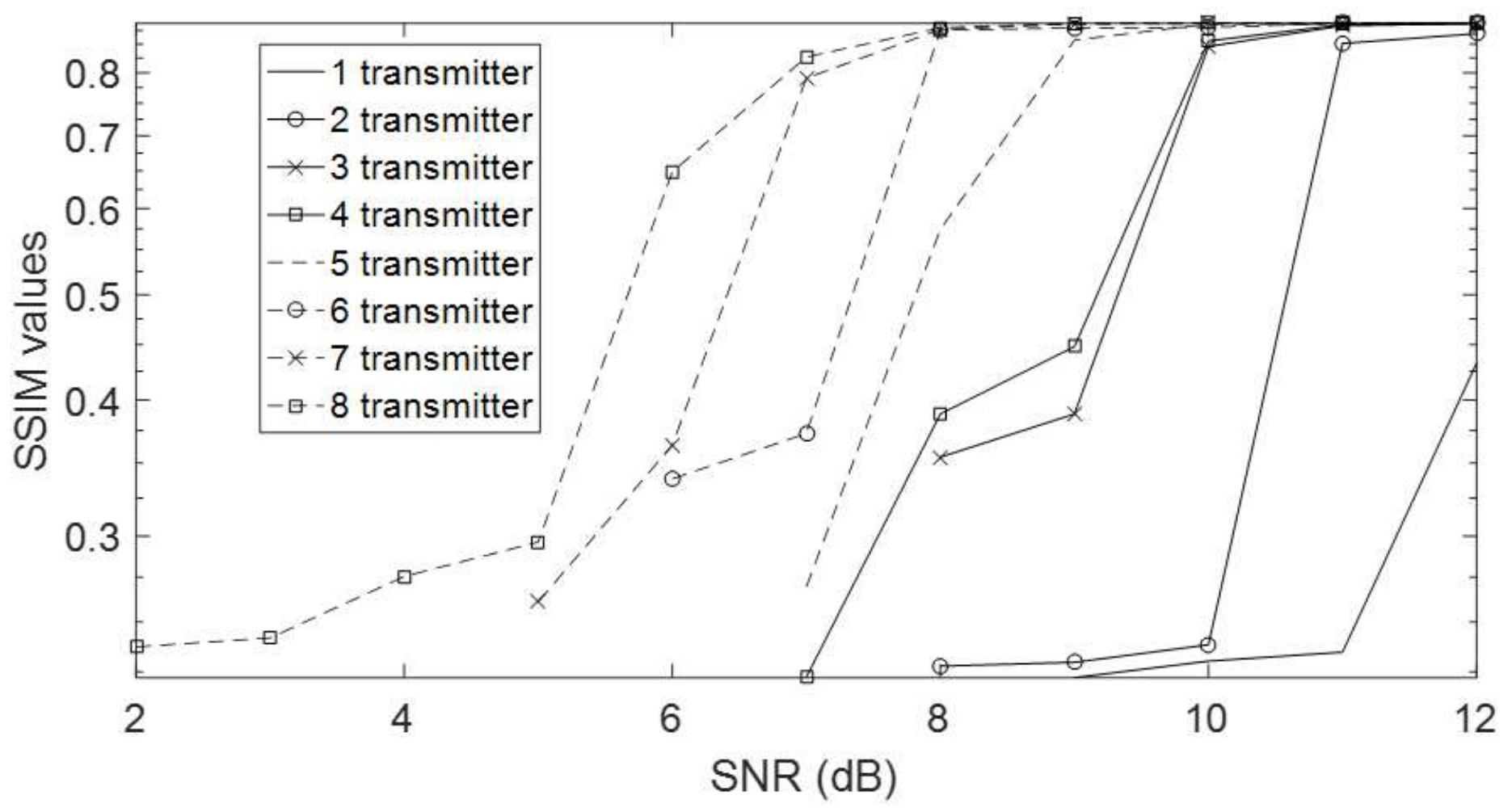

Figure 9

SSIM values for video transmission over VLC channel for different SNR values and number of transmitters 